\title{
Travelling front solutions arising in the chemotaxis-growth model
}

\author{
MitsuO FUNAKI ${ }^{\dagger}$ \\ Department of Mathematics, Hiroshima National College of Maritime Technology, \\ 4272-1 Higashino, Toyota-gun, Hiroshima, 725-0200, Japan \\ MASAYASU MiMURA \\ Department of Mathematics, School of Science and Technology, \\ Meiji Institute for Mathematical Science, Meiji University, \\ Higashimita, Tamaku, Kawasaki, 214-8571, Japan \\ AND \\ TOHRU TSUJIKAWA ${ }^{\S}$ \\ Faculty of Engineering, Miyazaki University, \\ Miyazaki, 889-2192, Japan
}

[Received 20 July 2005 and in revised form 5 January 2006]

\begin{abstract}
We consider a bistable reaction-diffusion-advection system describing the growth of biological individuals which move by diffusion and chemotaxis. We use the singular limit procedure to study the dynamics of growth patterns arising in this system. It is shown that travelling front solutions are transversally stable when the chemotactic effect is weak and, when it becomes stronger, they are destabilized. Numerical simulations reveal that the destabilized solution evolves into complex patterns with dynamic network-like structures.
\end{abstract}

Keywords: Chemotaxis; travelling front; singular perturbation; interfacial stability.

\section{Introduction}

Some biological individuals have a tendency to move preferentially toward higher concentrations of chemicals in their environment, which is called chemotaxis. It is experimentally observed, for instance, that bacteria called E.coli, which move by not only diffusion but also chemotaxis and grow by performing cell-division, to exhibit complex spatio-temporal colony patterns (Budrene and Berg [4, 5]).

For theoretical understanding of such chemotactic growth patterns, several continuum as well as discrete models have been proposed so far (see Woodward et al. [25], Brenner et al. [3], Stevens [19], Ezoe et al. [6], Kawasaki and Shigesada [12], for instance). In the previous paper (Mimura and Tsujikawa [14]), we considered a chemotaxis-growth model to investigate the influence of the chemotactic effect on growth patterns under the special situation where nutrients are constantly supplied. Let the density of biological individuals be $u(t, \mathbf{x})$ and the concentration of a chemical

\footnotetext{
${ }^{\dagger}$ E-mail: funaki@hiroshima-cmt.ac.jp

E-mail: mimura@math.meiji.ac.jp

${ }^{\S}$ E-mail: tujikawa@cc.miyazaki-u.ac.jp
} 
attractant be $v(t, \mathbf{x})$ at time $t$ and position $\mathbf{x}$ in the plane $\mathbb{R}^{2}$. The model is described by

$$
\left\{\begin{array}{l}
\frac{\partial u}{\partial t}=d_{u} \Delta u-\nabla(u \nabla \chi(v))+f(u), \\
\frac{\partial v}{\partial t}=d_{v} \Delta v+\alpha u-\beta v,
\end{array} \quad t>0, \mathbf{x} \in \mathbb{R}^{2},\right.
$$

where the migration of individuals consists of two effects, "randomly walking" by diffusion and "directed movements" by chemotaxis. $d_{u}$ and $d_{v}$ are the diffusion rates of $u$ and $v$, respectively. $\chi(v)$ is the chemotactic sensitivity function of the chemical attractant, satisfying $\chi^{\prime}(v)>0$ for $v>0$, in the sense that the flux rate of individuals is the response to its chemical gradient distributed in space. Several plausible forms of $\chi(v)$ have been proposed (Ford and Lauffenburger [9]). A simple example is $\chi(v)=k v$ with a constant $k>0$. The growth term $f(u)$ takes the form $f(u)=$ $(g(u)-\delta) u$ with growth rate $g(u)$ and degradation rate $\delta$ modelling the stress due to waste products (see [22], for instance). If $g(u)$ includes the Allee effect (see [16], for instance), the functional form of $g(u)$ is threshold-like. For suitable $\delta$, we may assume $f(u)$ to have a cubic-like nonlinearity, that is, $f(u)$ satisfies $f(0)=f\left(u_{*}\right)=f\left(u^{*}\right)=0$ with two constants $u_{*}$ and $u^{*}\left(0<u_{*}<u^{*}\right)$ where $f^{\prime}(0)<0$ and $f^{\prime}\left(u^{*}\right)<0$. For the chemical attractant $v, \alpha$ is the production rate and $\beta$ is the degradation rate, which are both positive constants.

For the system 1.1 we consider the situation where (i) the chemical attractant diffuses much faster than the movement of individuals; (ii) individuals mainly move by chemotaxis rather than diffusion. In order to model this situation, we conveniently introduce a small parameter $\varepsilon>0$ to rewrite 1.1 in the following form:

$$
\left\{\begin{array}{l}
\frac{\partial u}{\partial t}=\varepsilon^{2} \Delta u-\varepsilon \nabla(u \nabla \chi(v))+f(u), \\
\frac{\partial v}{\partial t}=\Delta v+u-\gamma v,
\end{array} \quad t>0, \mathbf{x} \in \mathbb{R}^{2}\right.
$$

where $\gamma$ is a positive constant and $f(u)$ has three zeros $0, a$ and $1(0<a<1)$. Hereafter, we simply specify $f(u)$ as $f(u)=u(1-u)(u-a)$ with $0<a<1$. Note that 1.2 has three spatially constant equilibria $(u, v)=(0,0),(a, a / \gamma)$ and $(1,1 / \gamma)$ for which $(0,0)$ and $(1,1 / \gamma)$ are both stable, while $(a, a / \gamma)$ is unstable, that is, 1.2$)$ is a bistable system. For the boundary condition to (1.2), it is biologically natural to impose

$$
\lim _{|\mathbf{x}| \rightarrow \infty}(u, v)(t, \mathbf{x})=(0,0), \quad t>0 .
$$

If there is no chemotactic effect in the system, 1.2 with $(1.3)$ simply reduces to the following scalar bistable reaction-diffusion equation:

$$
\frac{\partial u}{\partial t}=\varepsilon^{2} \Delta u+f(u), \quad t>0, \mathbf{x} \in \mathbb{R}^{2}
$$

with the boundary condition

$$
\lim _{|\mathbf{x}| \rightarrow \infty} u(t, \mathbf{x})=0, \quad t>0 .
$$

The qualitative behaviour of solutions of (1.4), (1.5) has been intensively investigated by many authors (see Aronson and Weinberger [1, 2], for instance). Suppose that the initial data $u(0, \mathbf{x})$ is 
given such that the region where $u(0, \mathbf{x})>a$ is bounded and relatively large. Then the behaviour of solutions to (1.4) essentially consists of two stages: (i) There occur internal layers which separate $\mathbb{R}^{2}$ into two qualitatively different regions where $u$ nearly takes the value either 1 or 0 , and (ii) if $\int_{0}^{1} f(u) \mathrm{d} u>0$ (resp. $<0$ ), the region where $u(t, \mathbf{x})$ is nearly 1 (we may call it the aggregating region) expands (resp. shrinks) uniformly (Jones [10, 11]). Thus, in the absence of chemotaxis, the pattern dynamics is so simple that $u$ expands uniformly and its shape becomes asymptotically disklike. If $\varepsilon$ is sufficiently small, the behaviour of internal layers can be more precisely analyzed by using the singular limit analysis (de Mottoni and Schatzman [15]). In the limit $\varepsilon \downarrow 0$, the layers become interfaces, say $\Gamma(t)$, which indicate the boundary between two regions $\Omega_{1}(t)=\left\{\mathbf{x} \in \mathbb{R}^{2}\right.$ | $u(t, \mathbf{x})=1\}$ and $\Omega_{0}(t)=\left\{\mathbf{x} \in \mathbb{R}^{2} \mid u(t, \mathbf{x})=0\right\}$, and the time evolution of $\Gamma(t)$ is approximately described by

$$
V(t)=\varepsilon(c-\varepsilon \kappa(t)),
$$

where $V(t)$ is the normal velocity at $\Gamma(t)$, which is oriented from $\Omega_{1}(t)$ to $\Omega_{0}(t)$ and $\kappa(t)$ is the curvature at $\Gamma(t)$. Here $c$ is the velocity of the travelling front solution $u(x-c t)$ of the following 1-dimensional problem:

$$
\left\{\begin{array}{l}
u_{t}=u_{x x}+f(u), \quad t>0, x \in \mathbb{R} \\
u(t,-\infty)=1 \quad \text { and } \quad u(t, \infty)=0
\end{array}\right.
$$

If $\int_{0}^{1} f(u) \mathrm{d} u>0$ (resp. $<0$ ), we know $c>0$ (resp. $<0$ ) (see Fife and McLeod [8], for example). In particular, if the shape of $\Gamma(0)$ is given by a disk with radius $r_{0}, \Gamma(t)$ is also a disk with radius $r(t)$, satisfying the simple differential equation

$$
\dot{r}=\varepsilon(c-\varepsilon / r), \quad t>0,
$$

with $r(0)=r_{0}$.

In this paper, we assume $\int_{0}^{1} f(u) \mathrm{d} u>0$ (or $0<a<1 / 2$ ) to require the situation where the aggregating region uniformly expands in the absence of chemotactic effect, and study how this effect influences the expanding pattern. More precisely, we study the dependence of the functional forms of $\chi(v)$ on the stability of the expanding pattern. Let us first show some numerical computations for $[1.2),(1.3)$. To do it, we put $\chi(v)=k \chi_{0}(v)$ with $\max _{v>0} \chi_{0}^{\prime}(v)=1$, so that $k$ is a parameter to measure the intensity of the chemotactic effect. Let us specify $\chi_{0}(v)$ as $\chi_{0}(v)=8 v^{2} / 3\left(3+v^{2}\right)$ (see Schaaf [18], for instance). The first case is restricted to the radially symmetric situation with $|\mathbf{x}|=r$, that is, the initial condition is given by $u(0, r)=1$ for $0<r<r_{0}$ and $u(0, r)=0$ for $r_{0}<r$ with some constant $r_{0}$ and $v(0, r) \equiv 0$ for any $r>0$. Because of radial symmetry, the solution of $[1.2]$, (1.3) is represented as $(u, v)(t, r)$ where there occurs an internal layer in $u(t, r)$, whose location is described by a circle in such a way that $u$ takes the value nearly 1 inside and nearly 0 outside of the circle. When $k$ is small, one can easily expect by (1.7) that the circle of internal layer uniformly expands with asymptotically constant velocity where the velocity is slightly slower than the one in the case $k=0$. When $k$ increases, the influence of chemotaxis on the expanding circle becomes apparent so that there appears a disk-like equilibrium solution. When $k$ still increases, there is no more disk-like equilibrium solution, the initial circle shrinks and finally becomes extinct [14]. These phenomena clearly suggest that the chemotactic effect suppresses expanding of growth.

Next, we numerically consider the case where the initial shape of $u(0, x)$ is slightly deformed from the circle. When $k$ is small, the deformation instantly decays and the pattern recovers the circular shape. However, when $k$ increases, the situation changes. The initial circular shape is destabilized so that there appears a flower-like pattern (Figure 113). When $k$ increases further, the 

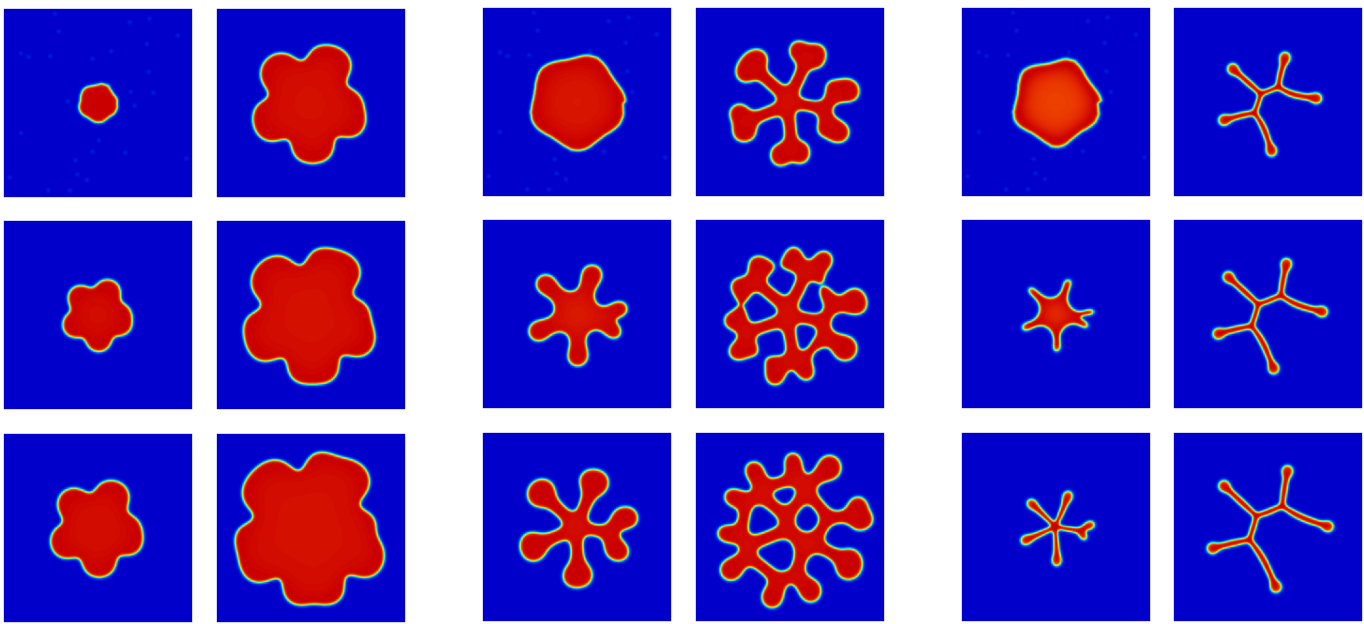

(b)
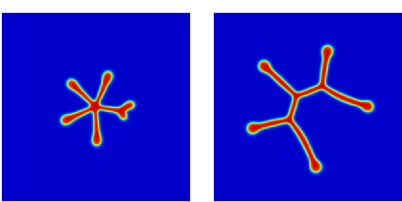

(c)

FIG. 1. Time evolution of $u(t, \mathbf{x})$ under non-radially symmetric initial conditions, where the curve the contour $C(t)=\{\mathbf{x} \in$ $\left.\mathbb{R}^{2} \mid u(t, \mathbf{x})=0.1\right\}$. Parameters are choosen as $\varepsilon=0.05, a=0.1$ and $\gamma=1.0$ and the system size is $20 \times 20$. (a) Formation of the flower-like pattern ( $k=1.3$ ): (i) $t=0$, (ii) $t=80$, (iii) $t=160$, (iv) $t=240$, (v) $t=320$, (vi) $t=400$. (b) Formation of network-like pattern ( $k=2.0$ ): (i) $t=0$, (ii) $t=100$, (iii) $t=200$ (iv) $t=300$, (v) $t=400$, (vi) $t=500$. (c) Formation of the finger-like pattern ( $k=5.0$ ): (i) $t=0$, (ii) $t=20$, (iii) $t=40$, (iv) $t=100$, (v) $t=200$, (vi) $t=300$.

circular shape is destabilized to be a star-like one with multi-branches and then the resulting pattern exhibits dynamic network-like structure through tip-splitting and coalescing processes (Figure 1p). When $k$ still increases further, the instability of the circular shape occurs, which is similar to the initiation in Figure $1 \mathrm{~b}$ and then each branch proceeds as if it were a 2-dimensional travelling finger wave. Also a triple junction phenomenon is observed (Figure 1p). These numerical results indicate that the chemotactic effect provides not only suppression of expanding but also shape-destabilization of patterns.

In order to analytically understand the dependence of the chemotactic effect on these properties, we consider the 1-dimensional travelling front solutions of (1.2) and their transversal stability in the strip domain $\Omega_{\ell}=\left\{(x, y) \in \mathbb{R}^{2} \mid-\infty<x<\infty, 0<y<\ell\right\}$ with width $\ell>0$, where the boundary conditions are

$$
\begin{cases}(u, v)(t,-\infty, y)=(1,1 / \gamma), & t>0,0<y<\ell, \\ (u, v)(t,+\infty, y)=(0,0), & t>0,0<y<\ell,\end{cases}
$$

and

$$
\begin{cases}\left(\frac{\partial u}{\partial y}, \frac{\partial v}{\partial y}\right)(t, x, 0)=(0,0), & t>0,-\infty<x<+\infty, \\ \left(\frac{\partial u}{\partial y}, \frac{\partial v}{\partial y}\right)(t, x, \ell)=(0,0), \quad t>0,-\infty<x<+\infty .\end{cases}
$$

This paper is organized as follows: In Section 2, we apply the singular perturbation method to (1.2) with a sufficiently small $\varepsilon>0$ and show the existence of 1-dimensional travelling front solutions $\left(U^{\varepsilon}, V^{\varepsilon}\right)(z)(z=x-\varepsilon \theta(\varepsilon) t)$ with velocity $\varepsilon \theta(\varepsilon)$. The profiles of $\left(U^{\varepsilon}, V^{\varepsilon}\right)(z)$ are shown 


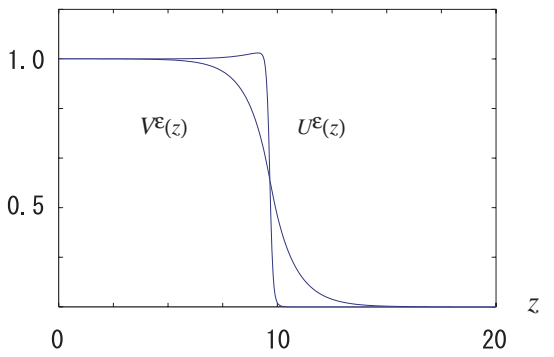

FIG. 2. Spatial profiles of the travelling front solution $\left(U^{\varepsilon}, V^{\varepsilon}\right)(z)$, where the parameters are as in Figure 1 except $k=1.0$.

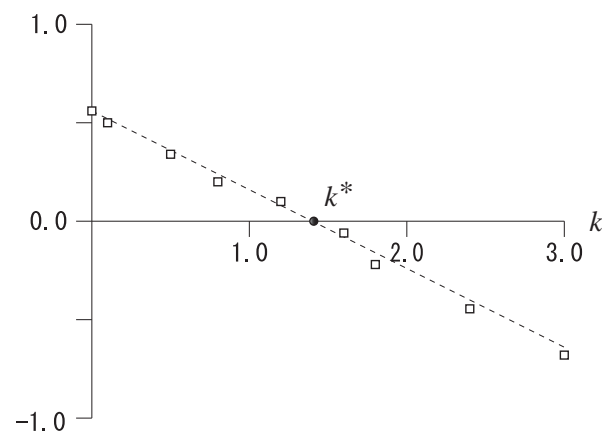

FIG. 3. Dependence of the velocity $\theta^{*}(k)$ on $k$ for a travelling front solution where $\square$ means the velocity given by 2.11]. The parameters except for $k$ are as in Figure 1

in Figure 2, they demonstrate that $V^{\varepsilon}(z)$ is smooth and $U^{\varepsilon}(z)$ possesses a single internal layer, which becomes a sharp interface as $\varepsilon \downarrow 0$. As for the dependence of $k$ on the velocity $\theta(\varepsilon)$, it is shown that $\theta(\varepsilon)$ is decreasing with $k$ and there is $k^{*}(\varepsilon)$ with $\lim _{\varepsilon \downarrow 0} k^{*}(\varepsilon)=k^{*}=2 \sqrt{\gamma} c / \chi_{0}^{\prime}(1 / 2 \gamma)$ such that $\theta(\varepsilon)>0$ for $0<k<k^{*}(\varepsilon)$, while $\theta(\varepsilon)<0$ for $k^{*}(\varepsilon)<k$ (Figure 3). Consequently, this result indicates that chemotaxis suppresses expanding of patterns.

In Section 3, we use the singular limit analysis as $\varepsilon \downarrow 0$ to study the transversal stability of the travelling front solutions $\left(U^{\varepsilon}, V^{\varepsilon}\right)(z)$ in the strip domain $\Omega_{\ell}$. Our result (Theorem 2) reveals that the transversal stability crucially depends on the sign of $\chi_{0}^{\prime \prime}\left(v_{\mathrm{I}}\right)$ where $v_{\mathrm{I}}$ is the value of the 1dimensional travelling front solution $V^{\varepsilon}(z)$ at the interfacial point, which is obtained by taking the limit $\varepsilon \downarrow 0$. If $\chi_{0}^{\prime \prime}\left(v_{\mathrm{I}}\right) \leqslant 0$, the solution is stable for any $k>0$ and $\ell>0$. However, if $\chi_{0}^{\prime \prime}\left(v_{\mathrm{I}}\right)>0$, the stability depends on the value of $k$. When $k$ is small, travelling front solutions are stable for any fixed $\ell>0$, while they are destabilized when $k$ increases. In order to show the stability property, we consider the distribution of eigenvalues of the linearized eigenvalue problem of $[1.2],[1.8],[1.9]$ around the planar travelling front solution, depending on the parameters $k$ and $\ell$. It should be noted that the fastest growing mode, say $m$, of the perturbations given in the planar interface is $O\left(\varepsilon^{-1 / 3}\right)$ for sufficiently small $\varepsilon>0$, that is, the fastest growing wavelength $2 \ell / m$ is $O\left(\varepsilon^{1 / 3}\right)$. This indicates that the destabilized pattern in short time does not depend on the width $\ell$ but on the smallness of $\varepsilon$. We remark that this behaviour is also observed in reaction-diffusion systems with activator-inhibitor interaction [21]. In Section 4, we give some remarks on our results. Finally, the Appendix is devoted to the proofs of the lemmas used in Section 3. 
The following function spaces are used in this paper:

$$
\begin{aligned}
C_{\text {unif }}(\mathbb{R}) & \equiv\{u \mid u \text { is bounded and uniformly continuous on } \mathbb{R}\}, \\
C_{\text {unif }}^{2}(\mathbb{R}) & \equiv\left\{u \in C^{2}(\mathbb{R}) \mid u(x), \frac{\mathrm{d}}{\mathrm{d} x} u(x), \frac{\mathrm{d}^{2}}{\mathrm{~d} x^{2}} u(x) \in C_{\text {unif }}(\mathbb{R})\right\}, \\
L_{\mu}^{2}(\mathbb{R}) & \equiv\left\{u \in L^{2}(\mathbb{R}) \mid\|u\|_{L_{\mu}^{2}} \equiv\left(\int_{\mathbb{R}}\left|e^{\mu|x|} u(x)\right|^{2} \mathrm{~d} x\right)^{1 / 2}<\infty\right\}, \\
H^{s}(\mathbb{R}) & \equiv \text { the interpolation space }\left[H^{1}(\mathbb{R}), L^{2}(\mathbb{R})\right]_{1-s} \text { for } 0<s<1, \\
H^{-1}(\mathbb{R}) & \equiv \text { the dual space of } H^{1}(\mathbb{R}), \quad H^{-s}(\mathbb{R}) \equiv \text { the dual space of } H^{s}(\mathbb{R}),
\end{aligned}
$$

and $C_{\text {c.u. }}^{2}(\mathbb{R})$ indicates uniform convergence on any compact subset of $\mathbb{R}$ with respect to $C^{2}(\mathbb{R})$ norm.

\section{Travelling front solutions}

We will briefly demonstrate how 1-dimensional travelling front solutions of $(1.2),(1.8)$ can be constructed, by using the well known singular perturbation methods. By introducing the travelling coordinate $z=x-\varepsilon \theta t$ with velocity $\varepsilon \theta$ in $[1.2$, one finds that the travelling front solution $(u, v)(z)$ satisfies the following system:

$$
\left\{\begin{array}{l}
0=\varepsilon^{2} u_{z z}+\varepsilon \theta u_{z}-\varepsilon k\left[u \chi_{0}^{\prime}(v) v_{z}\right]_{z}+f(u), \quad z \in \mathbb{R}, \\
0=v_{z z}+\varepsilon \theta v_{z}+u-\gamma v,
\end{array}\right.
$$

with the boundary conditions

$$
(u, v)(-\infty)=(1,1 / \gamma) \text { and }(u, v)(+\infty)=(0,0) .
$$

We first construct the outer and inner approximate solutions of 2.1 with 2.2, taking the limit $\varepsilon \downarrow 0$.

\subsection{Outer and inner solutions}

By putting $\varepsilon=0$ in 2.1), the lowest outer solution $\left(u^{0}, v^{0}\right)$ of 2.1 satisfies

$$
\left\{\begin{array}{l}
0=f(u), \\
0=v_{z z}+u-\gamma v, \quad z \in \mathbb{R} .
\end{array}\right.
$$

From the first equation of 2.3 with the boundary conditions 2.2 , we may take $u^{0}(z)$ as

$$
u^{0}(z)= \begin{cases}0 & (z>0) \\ 1 & (z<0)\end{cases}
$$

Substituting it into the second equation of 2.3 , we obtain $v^{0}(z)$ as

$$
v^{0}(z)= \begin{cases}\frac{1}{2 \gamma} \exp (-\sqrt{\gamma} z) & (z>0), \\ \frac{1}{\gamma}-\frac{1}{2 \gamma} \exp (\sqrt{\gamma} z) & (z<0),\end{cases}
$$


which belongs to $C^{1}(\mathbb{R}) .\left(u^{0}, v^{0}\right)(z)$ is called an outer solution of 2.1, , 2.2 in $\mathbb{R}$. Since $u^{0}(z)$ is discontinuous at $z=0$, it is not an approximate solution of (2.1) in a neighbourhood of $z=0$, so that we have to look for another approximate solution there. In order to construct an approximate solution in a neighbourhood of $z=0$, we introduce the usual stretched variable $\xi=z / \varepsilon$ and rewrite 2.1) as

$$
\left\{\begin{array}{l}
0=\tilde{u}_{\xi \xi}+\left\{\theta-k \chi_{0}^{\prime}(\tilde{v}) \tilde{v}_{z}\right\} \tilde{u}_{\xi}-\varepsilon k\left\{\chi_{0}^{\prime}(\tilde{v}) \tilde{v}_{z}\right\}_{z} \tilde{u}+f(\tilde{u}), \quad \xi \in \mathbb{R}, \\
0=\tilde{v}_{\xi \xi}+\varepsilon^{2}\left\{\theta \tilde{v}_{\xi}+\tilde{u}-\gamma \tilde{v}\right\},
\end{array}\right.
$$

where $(\tilde{u}, \tilde{v})(\xi)=(u, v)(\varepsilon \xi)$. Putting $\varepsilon=0$ in 2.6 and noting $v^{0}(0)=1 / 2 \gamma$, we obtain

$$
\left\{\begin{array}{l}
0=\tilde{u}_{\xi \xi}+\left\{\theta-k \chi_{0}^{\prime}(\tilde{v}) \tilde{v}_{z}\right\} \tilde{u}_{\xi}+f(\tilde{u}), \quad \xi \in \mathbb{R}, \\
0=\tilde{v}_{\xi \xi},
\end{array}\right.
$$

with the boundary conditions

$$
\left\{\begin{array}{l}
\tilde{u}(-\infty)=1, \quad \tilde{u}(+\infty)=0 \\
\tilde{v}( \pm \infty)=\frac{1}{2 \gamma}
\end{array}\right.
$$

Since the second equation of 2.7) with (2.8) leads to $\tilde{v}(\xi) \equiv 1 / 2 \gamma$, the first equation of 2.77 is

$$
0=\tilde{u}_{\xi \xi}+\left\{\theta+\frac{k}{2 \sqrt{\gamma}} \chi_{0}^{\prime}\left(\frac{1}{2 \gamma}\right)\right\} \tilde{u}_{\xi}+f(\tilde{u}) .
$$

In order to solve the equation (2.9) with (2.8), we need the following lemma:

LEMmA 1 (Fife and McLeod [8]) For any fixed $\zeta \in(0,1)$, there exists a unique $c$ such that the following problem has a unique decreasing solution $W(\xi ; c)$ :

$$
\left\{\begin{array}{l}
0=W_{\xi \xi}+c W_{\xi}+f(W), \quad \xi \in \mathbb{R} \\
W(-\infty)=1, \quad W(+\infty)=0, \quad W(0)=\zeta
\end{array}\right.
$$

Furthermore, if $\int_{0}^{1} f(u) \mathrm{d} u>0$ (resp. $\left.<0\right)$, then $c>0$ (resp. $\left.<0\right)$.

Lemma 1 indicates that a solution $\tilde{u}$ of 2.9 with 2.8 is given by $\tilde{u}(\xi)=W(\xi)$ with $\theta^{*}(k)=$ $c-k \chi_{0}^{\prime}(1 / 2 \gamma) / 2 \sqrt{\gamma} ;(\tilde{u}, \tilde{v})(z / \varepsilon)$ is called an inner solution in a neighbourhood of $z=0$.

\subsection{Existence of 1-dimensional travelling front solutions}

By matching the outer and inner solutions obtained above, travelling front solutions of the problem [2.1], 2.2) can be constructed (see Fife [7], Mimura et al. [13], for example). The result is stated as follows:

THEOREm 1 Fix $k>0$ arbitrarily. There is $\varepsilon_{0}>0$ such that for any $\varepsilon \in\left(0, \varepsilon_{0}\right)$, 2.1), 2.2) has a solution $\left(U^{\varepsilon}, V^{\varepsilon}\right)(z)$ with $\theta=\theta(\varepsilon ; k)$ satisfying

$$
\lim _{\varepsilon \downarrow 0} \theta(\varepsilon ; k)=\theta^{*}(k)=c-\frac{k}{2 \sqrt{\gamma}} \chi_{0}^{\prime}\left(\frac{1}{2 \gamma}\right) .
$$


The solution $\left(U^{\varepsilon}, V^{\varepsilon}\right)$ is represented as

$$
U^{\varepsilon}(z)=W(z / \varepsilon)+p^{\varepsilon}(z) \text { and } \quad V^{\varepsilon}(z)=v^{0}(z)+q^{\varepsilon}(z)
$$

where $\left(p^{\varepsilon}, q^{\varepsilon}\right)$ is an error term for small $\varepsilon>0$ in view of the following convergences:

$$
\begin{cases}\lim _{\varepsilon \downarrow 0} U^{\varepsilon}(z)=u^{0}(z) & \text { uniformly in }(-\infty,-\delta) \cup(\delta,+\infty), \\ \lim _{\varepsilon \downarrow 0} V^{\varepsilon}(z)=v^{0}(z) & \text { uniformly in } \mathbb{R},\end{cases}
$$

with any small constant $\delta>0$ and

$$
\begin{cases}\lim _{\varepsilon \downarrow 0} \tilde{u}^{\varepsilon}(\xi)=W(\xi) & \text { in } C_{\text {c.u. }}^{2}(\mathbb{R}), \\ \lim _{\varepsilon \downarrow 0} \tilde{v}^{\varepsilon}(\xi)=\frac{1}{2 \gamma} & \text { in } C_{\text {c.u. }}^{2}(\mathbb{R}),\end{cases}
$$

where $\left(\tilde{u}^{\varepsilon}, \tilde{v}^{\varepsilon}\right)(\xi)=\left(U^{\varepsilon}, V^{\varepsilon}\right)(\varepsilon \xi)$.

The profile of $\left(U^{\varepsilon}, V^{\varepsilon}\right)(z)$ is shown in Figure 2. By 2.11, we immediately obtain

Proposition 1 (i) If $\int_{0}^{1} f(u) \mathrm{d} u \leqslant 0(c \leqslant 0)$, then $\theta^{*}(k)<0$ for any $k>0$.

(ii) If $\int_{0}^{1} f(u) \mathrm{d} u>0(c>0)$, then there is the critical value $k^{*}=2 \sqrt{\gamma} c / \chi_{0}^{\prime}(1 / 2 \gamma)$ such that $\theta^{*}(k)>0$ for any $0<k<k^{*}$, while $\theta^{*}(k)<0$ for any $k>k^{*}$.

For the case (ii) of Proposition 1 , the dependence of $\theta^{*}(k)$ and $\theta(\varepsilon ; k)$ on $k$ is shown in Figure 3 . The uniqueness of the travelling front solution of 2.1, 2.2 has been numerically confirmed, although it is still unsolved. Since $\varepsilon c=\varepsilon \theta(\varepsilon ; 0)$ is the velocity of the travelling front solution of 2.1), 2.2) in the absence of chemotaxis $(k=0), 2.11$ indicates that chemotaxis suppresses the expansion of patterns.

\section{Transversal stability of travelling front solutions}

In Section 1, by numerical simulations, we found that chemotaxis effects destabilization of patterns. In order to show this theoretically, we focus on the travelling front solutions $\left(U^{\varepsilon}, V^{\varepsilon}\right)(z)$ and study their transversal stability in a strip domain $\Omega_{\ell}=\mathbb{R} \times(0, \ell)$. We first define the linearized stability of travelling front solutions of (1.2), (1.8), 1.9) as follows:

DEFINITION A travelling front solution of (1.2), 1.8, 1.9 is transversally stable except for translational invariance in $x$ if and only if zero is a simple eigenvalue of the eigenvalue problem associated with the linearized system of $(1.2),(1.8),(1.9)$ around the travelling front solution and the remaining spectrum is contained in a closed sector lying in the left complex half-plane (see Volpert et al. [24], for instance). The travelling front solution is unstable if it is not stable.

We only study the distribution of eigenvalues of the eigenvalue problem of the linearized system of 1.2, 1.8, 1.9 around the travelling front solutions $\left(\hat{u}^{\varepsilon}, \hat{v}^{\varepsilon}\right)(z, y)=\left(U^{\varepsilon}, V^{\varepsilon}\right)(z)$ with velocity $\varepsilon \theta(\varepsilon ; k)$ in the strip domain $\Omega_{\ell}$. Here we write $\theta(\varepsilon ; k)$ as $\theta(\varepsilon)$ for simplicity. The eigenvalue 
problem for $(p, q)(z, y)$ associated with [1.2), (1.8), 1.9$)$ is given by

$$
\left\{\begin{array}{rl}
\lambda p= & {\left[\varepsilon^{2}\left(\frac{\partial^{2}}{\partial z^{2}}+\frac{\partial^{2}}{\partial y^{2}}\right)+\varepsilon \theta(\varepsilon) \frac{\partial}{\partial z}-\varepsilon k\left\{\left(\chi_{0}^{\prime}\left(\hat{v}^{\varepsilon}\right) \hat{v}_{z}^{\varepsilon}\right) \frac{\partial}{\partial z}\right.\right.} \\
& \left.\left.+\left(\chi_{0}^{\prime}\left(\hat{v}^{\varepsilon}\right) \hat{v}_{z}^{\varepsilon}\right)_{z}\right\}+f^{\prime}\left(\hat{u}^{\varepsilon}\right)\right] p-\varepsilon k\left[\hat{u}^{\varepsilon} \chi_{0}^{\prime}\left(\hat{v}^{\varepsilon}\right)\left(\frac{\partial^{2}}{\partial z^{2}}+\frac{\partial^{2}}{\partial y^{2}}\right)\right. \\
& \left.+\left\{\hat{u}^{\varepsilon} \chi_{0}^{\prime \prime}\left(\hat{v}^{\varepsilon}\right) \hat{v}_{z}^{\varepsilon}+\left(\hat{u}^{\varepsilon} \chi_{0}^{\prime}\left(\hat{v}^{\varepsilon}\right)\right)_{z}\right\} \frac{\partial}{\partial z}+\left(\hat{u}^{\varepsilon} \chi_{0}^{\prime \prime}\left(\hat{v}^{\varepsilon}\right) \hat{v}_{z}^{\varepsilon}\right)_{z}\right] q, \\
\lambda q= & p+\left\{\left(\frac{\partial^{2}}{\partial z^{2}}+\frac{\partial^{2}}{\partial y^{2}}\right)+\varepsilon \theta(\varepsilon) \frac{\partial}{\partial z}-\gamma\right\} q,
\end{array} \quad(z, y) \in \Omega_{\ell},\right.
$$

with the boundary conditions

$$
\left\{\begin{array}{lll}
p( \pm \infty, y)=0, & q( \pm \infty, y)=0, & 0<y<\ell, \\
\frac{\partial p}{\partial y}(z, 0)=0, & \frac{\partial q}{\partial y}(z, 0)=0, & z \in \mathbb{R}, \\
\frac{\partial p}{\partial y}(z, \ell)=0, & \frac{\partial q}{\partial y}(z, \ell)=0, & z \in \mathbb{R} .
\end{array}\right.
$$

For $(p, q)(z, y) \in L^{2}\left(\Omega_{\ell}\right) \times L^{2}\left(\Omega_{\ell}\right)$, we define $\left(p_{m}, q_{m}\right)(z)(z \in \mathbb{R}, m=0,1,2, \ldots)$ by

$$
p_{m}(z)=\int_{0}^{\ell} p(z, y) Y_{m}(y) \mathrm{d} y, \quad q_{m}(z)=\int_{0}^{\ell} q(z, y) Y_{m}(y) \mathrm{d} y,
$$

where

$$
Y_{m}(y)= \begin{cases}\frac{1}{\sqrt{\ell}} & \text { for } m=0, \\ \sqrt{\frac{2}{\ell}} \cos \left(\frac{m \pi y}{\ell}\right) & \text { for } m \geqslant 1 .\end{cases}
$$

Then $(p, q)(z, y)$ is expanded as

$$
p(z, y)=\sum_{m=0}^{\infty} p_{m}(z) Y_{m}(y), \quad q(z, y)=\sum_{m=0}^{\infty} q_{m}(z) Y_{m}(y)
$$

in $L^{2}\left(\Omega_{\ell}\right)$. It thus follows from 3.1 that the equations for $\left(p_{m}, q_{m}\right)(z)$ with $\omega=m \pi / \ell$ are given by

$$
\left\{\begin{array}{l}
\lambda p_{m}=\left(L^{\varepsilon}-\varepsilon^{2} \omega^{2}\right) p_{m}-N^{\varepsilon, \omega} q_{m}, \quad z \in \mathbb{R} \\
\lambda q_{m}=p_{m}+\left(M^{\varepsilon}-\omega^{2}\right) q_{m}, \\
p_{m}, q_{m} \in C_{\mathrm{unif}}^{2}(\mathbb{R})
\end{array}\right.
$$


where

$$
\begin{aligned}
L^{\varepsilon} & \equiv \varepsilon^{2} \frac{\mathrm{d}^{2}}{\mathrm{~d} z^{2}}+\varepsilon\left\{\theta(\varepsilon)-k \chi_{0}^{\prime}\left(V^{\varepsilon}\right) V_{z}^{\varepsilon}\right\} \frac{\mathrm{d}}{\mathrm{d} z}-\varepsilon k\left\{\chi_{0}^{\prime}\left(V^{\varepsilon}\right) V_{z}^{\varepsilon}\right\}_{z}+f^{\prime}\left(U^{\varepsilon}\right), \\
N^{\varepsilon, \omega} & \equiv \varepsilon k\left[U^{\varepsilon} \chi_{0}^{\prime}\left(V^{\varepsilon}\right)\left\{\frac{\mathrm{d}^{2}}{\mathrm{~d} z^{2}}-\omega^{2}\right\}+\left\{U_{z}^{\varepsilon} \chi_{0}^{\prime}\left(V^{\varepsilon}\right)+2 U^{\varepsilon} \chi_{0}^{\prime \prime}\left(V^{\varepsilon}\right) V_{z}^{\varepsilon}\right\} \frac{\mathrm{d}}{\mathrm{d} z}+\left\{U^{\varepsilon} \chi_{0}^{\prime \prime}\left(V^{\varepsilon}\right) V_{z}^{\varepsilon}\right\}_{z}\right], \\
M^{\varepsilon} & \equiv \frac{\mathrm{d}^{2}}{\mathrm{~d} z^{2}}+\varepsilon \theta(\varepsilon) \frac{\mathrm{d}}{\mathrm{d} z}-\gamma .
\end{aligned}
$$

For the eigenvalues of 3.2 , we obtain the following two lemmas:

LEMma 2 There is a constant $C_{1}>0$ and for any given $d>0$ there is $\varepsilon_{1}>0$ such that any eigenvalue $\lambda \in \mathbb{C}$ of (3.2) satisfies either $\operatorname{Re} \lambda<-C_{1}$ or

$$
\left|\varepsilon^{2} \omega^{2}+\lambda\right|<d \quad \text { for } 0<\varepsilon<\varepsilon_{1} \text { and } \omega>0 .
$$

This lemma indicates that the distribution of eigenvalues of $(3.2)$ is divided into two classes as $\varepsilon \downarrow 0$ : one class is the distribution of eigenvalues of the order $O(1)$ which have negative real part and the other is of the order $o(1)$. Therefore, the distribution of the latter eigenvalues is critical for the stability of travelling front solutions. By (3.3), we may assume that there is a positive function $d(\varepsilon)$ with $\lim _{\varepsilon \downarrow 0} d(\varepsilon)=0$ such that

$$
\left|\varepsilon^{2} \omega^{2}+\lambda\right|<d(\varepsilon)
$$

For the eigenvalues of 3.2) satisfying (3.4), we have the following key lemma:

LEMMA 3 For sufficiently small $\varepsilon>0$, let $\lambda_{m}$ be an eigenvalue of (3.2) which satisfies (3.4). Then there exists a continuous function $\tau_{m}(\varepsilon, \ell, k)$ such that $\lambda_{m}=\varepsilon \tau_{m}(\varepsilon, \ell, k)$ satisfying

$$
\lim _{\varepsilon \downarrow 0}\left\{\tau_{m}(\varepsilon, \ell, k)-\tau_{m}^{*}(\varepsilon, \ell, k)\right\}=0
$$

with

$$
\tau_{m}^{*}(\varepsilon, \ell, k) \equiv-\varepsilon\left(\frac{m \pi}{\ell}\right)^{2}+\frac{k}{4 \sqrt{\gamma}}\left(\frac{1}{\sqrt{\gamma}}-\frac{1}{\sqrt{\gamma+(m \pi / \ell)^{2}}}\right) \chi_{0}^{\prime \prime}\left(\frac{1}{2 \gamma}\right) .
$$

When $m=0$, we know $\tau_{0}^{*}(\varepsilon, \ell, k)=0$, which corresponds to the zero eigenvalue of (3.2) with the eigenfunction $\left(U_{z}^{\varepsilon}, V_{z}^{\varepsilon}\right)(z)$ where $\left(U^{\varepsilon}, V^{\varepsilon}\right)(z)$ is the travelling front solution of $(1.2)$. Lemmas 2 and 3 are proved in the Appendix. By using these lemmas, we can easily arrive at the following theorem:

THEOREM 2 (i) When $\chi_{0}^{\prime \prime}(1 / 2 \gamma) \leqslant 0$, for any fixed $\ell>0$ and $k>0$, there is $\varepsilon_{0}>0$ such that if $0<\varepsilon<\varepsilon_{0}$, then $\lambda_{m}<0$ for any $m>0$, i. e. the travelling front solution is transversally stable. (ii) When $\chi_{0}^{\prime \prime}(1 / 2 \gamma)>0$, for any fixed $\ell>0$ and $k>0$, there is $\varepsilon_{0}(\ell, k)>0$ such that for any $0<\varepsilon<\varepsilon_{0}(\ell, k)$, the travelling front solution is transversally unstable.

It follows from Theorem 2 and $(2.13)$ that transversal stability of travelling front solutions depends on the sign of $\chi_{0}^{\prime \prime}\left(v_{\mathrm{I}}\right)$ where $v_{\mathrm{I}}$ is the value of $V(z)$ on the interface. In fact, when 


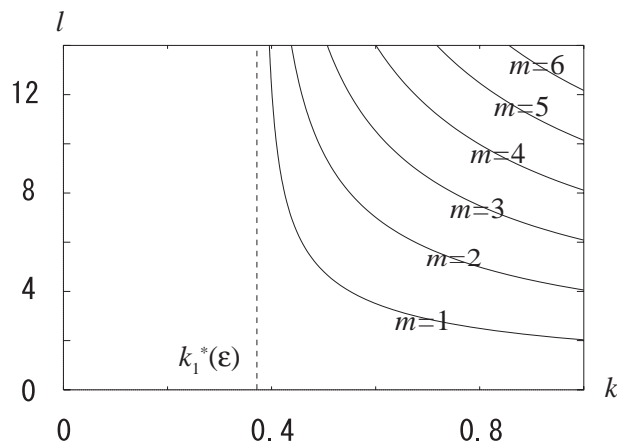

FIG. 4. Bifurcation curves $k=k_{m}(\varepsilon, \ell)(m=1,2, \ldots)$ of the flat travelling front solution where $\chi_{0}(v)=8 v^{2} / 3\left(3+v^{2}\right)$. The parameters except for $k$ are as in Figure 1 Here $m$ is the mode number of perturbations and $k_{1}^{*}(\varepsilon)=\lim _{\ell \rightarrow \infty} k_{1}(\varepsilon, \ell)$.

$\chi_{0}^{\prime \prime}\left(v_{\mathrm{I}}\right)>0$, the travelling front solution is destabilized as $k$ increases. Let $\ell$ be fixed and $\lambda_{m_{0}}(\varepsilon)$ be the largest eigenvalue. Then it follows from (3.5) that for sufficiently small $\varepsilon, m_{0}(\varepsilon)$ satisfies

$$
\left|\varepsilon^{1 / 3} m_{0}(\varepsilon)-\frac{\ell}{2 \pi}\left(\frac{k \chi_{0}^{\prime \prime}(1 / 2 \gamma)}{\sqrt{\gamma}}\right)^{1 / 3}\right|<\delta(\varepsilon),
$$

where $\delta(\varepsilon)$ is a positive function satisfying $\lim _{\varepsilon \downarrow 0} \delta(\varepsilon)=0$. Therefore, the fastest growth wavelength $\mu_{0}(\varepsilon)=2 \ell / m_{0}(\varepsilon)$ satisfies $\mu_{0}(\varepsilon)=O\left(\varepsilon^{1 / 3}\right)$ for sufficiently small $\varepsilon$. This implies that the wavelength $\mu_{0}(\varepsilon)$ does not depend on the width $\ell$ but on the smallness of $\varepsilon$, that is, when $\varepsilon$ is sufficiently small, any fine structure with wavelength which is $O\left(\varepsilon^{1 / 3}\right)$ appears in the first time scale.

Let us apply this theorem to two specific forms of $\chi_{0}(v)$. First, we take $\chi_{0}(v)=v$. Since $\chi_{0}^{\prime \prime}(v) \equiv 0$, the travelling front solution is always transversally stable for any $k>0$. We next take $\chi_{0}(v ; s)=8 \sqrt{3 s} v^{2} / 9\left(s+v^{2}\right)$, for which there is some value $s^{*}=3 / 4 \gamma^{2}$ such that $\chi_{0}^{\prime \prime}(1 / 2 \gamma ; s) \leqslant 0$ for $0<s \leqslant s^{*}$ and $\chi_{0}^{\prime \prime}(1 / 2 \gamma ; s)>0$ for $s^{*}<s$. For the latter case, the bifurcation curves of $\tau_{m}^{*}(\varepsilon, \ell, k)=0(m=1,2, \ldots)$ are drawn in the $(k, \ell)$-plane, as in Figure 4 This indicates the following:

(i) Let $k=k_{m}(\varepsilon, \ell)$ be a solution of $\tau_{m}^{*}(\varepsilon, \ell, k)=0(m=1,2, \ldots)$. Then $k_{1}(\varepsilon, \ell)<k_{2}(\varepsilon, \ell)$ $<\cdots$ for any fixed $\ell>0$.

(ii) There is the critical value

$$
k_{*}(\varepsilon, \ell)=4 \varepsilon \gamma \sqrt{\gamma+(\pi / \ell)^{2}}\left(\sqrt{\gamma}+\sqrt{\gamma+(\pi / \ell)^{2}}\right) / \chi_{0}^{\prime \prime}\left(\frac{1}{2 \gamma}\right)
$$

such that if $k<k_{*}(\varepsilon, \ell)$, then $\lambda_{m}<0$ for any $m>0$, while if $k_{*}(\varepsilon, \ell)<k$, then $\lambda_{m}>0$ for some $m>0$.

Figure 4 shows that for any fixed width $\ell>0$, the travelling front solution is transversally destabilized as $k$ increases. Let us show some numerical simulations for the problem (1.2), (1.8), (1.9) in $\Omega_{\ell}$ with suitably large $\ell>0$. We use $\chi_{0}(v ; s)$ and fix $\gamma=1$. Since $s^{*}=3 / 4$, we take $\chi_{0}(v ; 3)=8 v^{2} / 3\left(3+v^{2}\right)$. When $k$ is small, the travelling front solution is transversally stable (Figure 5a). When $k$ increases so that $k>k_{1}(\varepsilon, \ell)$, it becomes unstable (Figure 5b). When $k$ 
(a) (i)

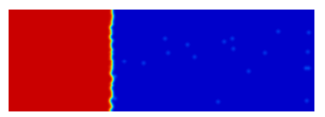

(ii)

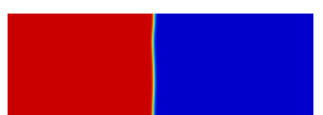

(iii)
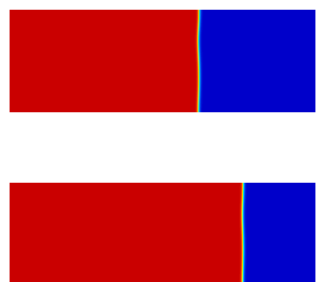

(c) (i)

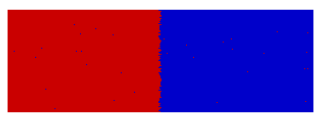

(ii)

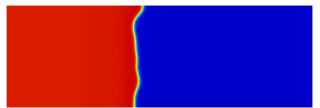

(iii)

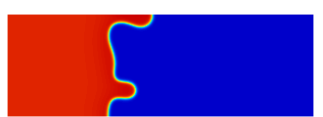

(iv)

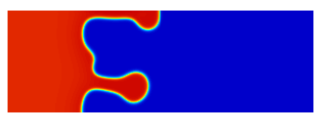

(v)

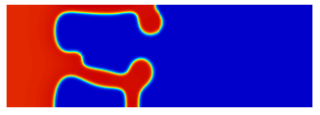

(vi)

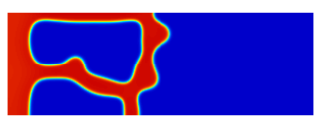

(b) (i)

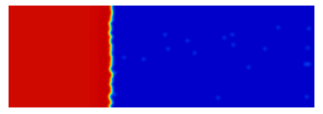

(ii)

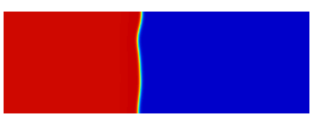

(iii)

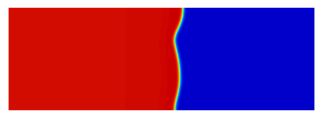

(iv)

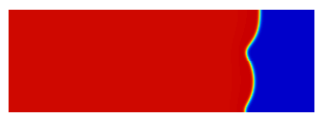

(d) (i)

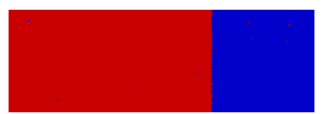

(ii)

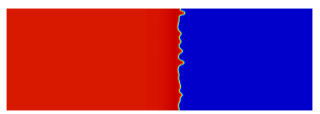

(iii)

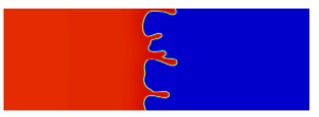

(iv)

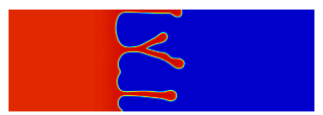

(v)

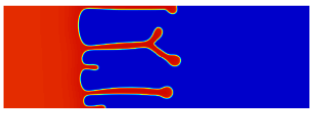

FIG. 5. Time evolution of $u(t, \mathbf{x})$, where the curves depict the contour $C(t)=\left\{\mathbf{x} \in \Omega_{\ell} \mid u(t, \mathbf{x})=0.1\right\}$, with the parameters being as in Figure 1 except for the system size $10 \times 30$. (a) Transversal stability of a perturbed travelling front solution ( $k=0.3$ ): (i) $t=0$, (ii) $t=100$, (iii) $t=200$, (iv) $t=300$. (b) Destabilized travelling front solution $(k=1.0)$ : (i) $t=0$, (ii) $t=200$, (iii) $t=400$, (iv) $t=600$. (c) Formation of the network-like pattern $(k=2.0$ ): (i) $t=0$, (ii) $t=100$, (iii) $t=200$, (iv) $t=300$, (v) $t=400$, (vi) $t=500$. (d) Formation of the finger-like pattern $(k=5.0$ ): (i) $t=0$, (ii) $t=200$, (iii) $t=400$, (iv) $t=600$, (v) $t=800$. 
increases further, the travelling front solution evolves into a complex pattern (Figure 55), where two features can be observed: one is tip-splitting to generate a branching pattern and the other is coalescing of these branches, by which network-like structures appear. When $k$ still increases further, the situation drastically changes. The destabilized travelling front solution exhibits a fingerlike pattern (Figure 5d). For this pattern, we note that the interfaces are initially destabilized with small wavelengths of the order $O\left(\varepsilon^{1 / 3}\right)$, as was noted, but in the next stage, this fine structure breaks and there appear finger-like branched patterns with width of the order $O(1)$.

\section{Concluding remarks}

The numerical simulations suggest that chemotaxis has two effects: one is suppression of expanding of patterns, the other is transversal destabilization of patterns. To understand these properties, we have studied the existence and transversal stability of 1-dimensional travelling front solutions in the strip domain. As was shown in Figure 3 , the velocity of travelling front solutions is positive for small $k$, while it is negative for large $k$. This clearly explains that the chemotactic effect inhibits the expansion of growth patterns. We have shown in Theorem 2 that transveral stability of travelling front solutions depends on the sign of $\chi_{0}^{\prime \prime}(1 / 2 \gamma)$ for the chemotactic sensitivity function $\chi_{0}(v)$. If $\chi_{0}^{\prime \prime}(1 / 2 \gamma)>0$, the travelling front solution is destabilized as $k$ increases. If $k$ is small, the travelling front solution is transversally stable. However, when $k$ increases, it becomes unstable through static

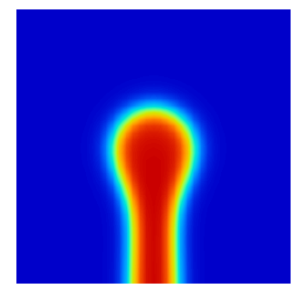

U

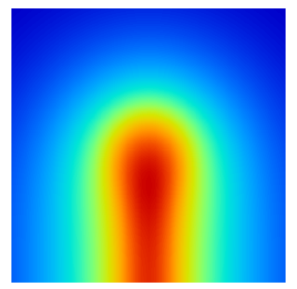

V

FIG. 6. 2-dimensional travelling finger-like solution with positive velocity, where the parameters are as in Figure 1 except for $k=5.0$ and the system size $3 \times 3$. The values of $u(t, \mathbf{x})$ and $v(t, \mathbf{x})$ become larger from outside to inside.

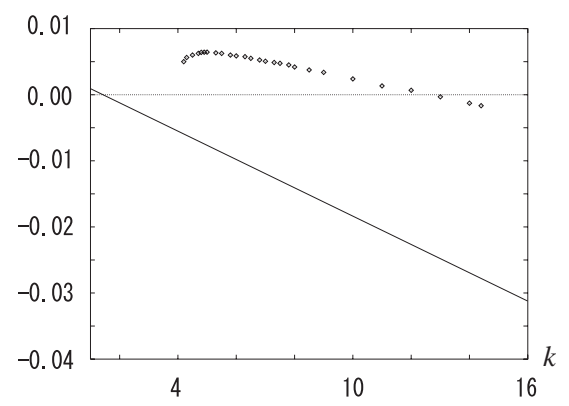

FIG. 7. $\diamond$ means the velocity of 2-dimensional travelling finger-like solutions, where the parameters are as in Figure 1 means the velocity $\theta^{*}(k)$ of 1 -dimensional travelling front solutions as $\varepsilon \downarrow 0$. 
(i)

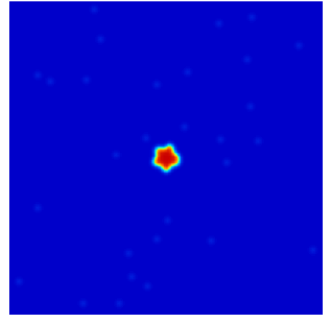

(ii)

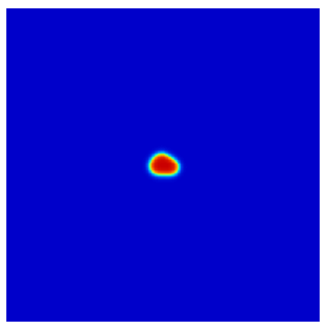

(iii)

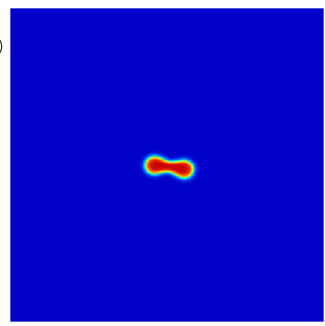

(iv)

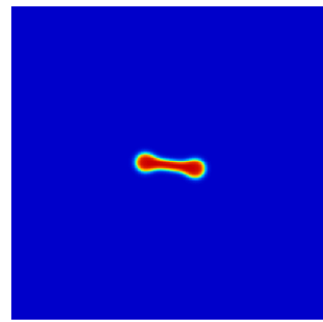

(v)

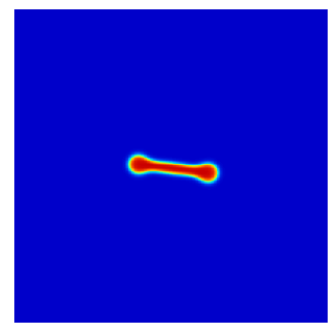

(vi)

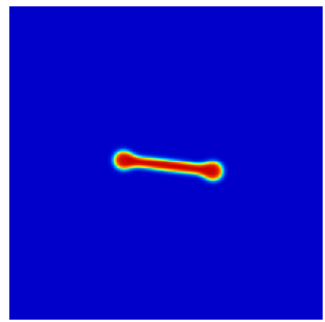

FIG. 8. Time evolution of $u(t, \mathbf{x})$ under non-radially symmetric initial conditions, where the curve is the contour $C(t)=$ $\left\{\mathbf{x} \in \mathbb{R}^{2} \mid u(t, \mathbf{x})=0.1\right\}$. Parameters are as in Figure 6 except the system size $20 \times 20$. (i) $t=0$, (ii) $t=100$, (iii) $t=200$, (iv) $t=300$, (v) $t=400$, (vi) $t=500$.

bifurcation. We discussed the stability analysis of planar travelling front solutions, depending on the functional form of $\chi(v)$. The resulting destabilized patterns are quite interesting. If $k$ increases further, the destabilized pattern exhibits tip-splitting and coalescing phenomena alternatively so that a network-like pattern appears, as in Figure 5. If $k$ still increases, it shrinks and generates branched patterns as in Figure 5 $\mathrm{d}$. This structure is similar to the one shown in Figure 11. We should note that each branch grows as if it were a 2-dimensional travelling finger-like solution which moves with constant velocity, which is numerically confirmed, as in Figure 6. The comparison between 1dimensional travelling front solution and a 2-dimensional travelling branch solution is demonstrated in Figure 7. We should remark that for relatively large $k$ the velocity of 1-dimensional travelling front solutions is negative, while the velocity of 2-dimensional travelling finger-like solutions is positive. For the possibility of existence of such 2-dimensional finger-like solutions, there are three plausible reasons by using the results obtained in [14], [23]: (i) The travelling finger-like solution in $\mathbb{R}^{2}$ is regarded as a heteroclinic orbit connecting two stable states; one is $(0,0)$ at $y=\infty$ and the other is $\left(U_{p}(x), V_{p}(x)\right)$ at $y=-\infty$ where $\left(U_{p}(x), V_{p}(x)\right)$ is the 1-dimensional stable equilibrium pulse solution. (ii) The equilibrium pulse solution is transversally stable in the strip domain. This suggests that the side parts of a finger stably exist. (iii) 2-dimensional disk-like equilibrium solutions 
are unstable under 2-mode disturbance. As in Figure 8 a disk-like equilibrium solution destabilizes to form a peanut shape. It seems that this is the onset of travelling finger-like solutions. The stability of 2-dimensional travelling finger-like solutions will be the subject of our future work.

\section{Acknowledgments}

We would like to thank the referees for their useful comments. The second and third authors are supported by Grant-in-Aid for Scientific Reserch (A) 08404005, 09354001 and (C) 17540125 by the Japan Society for Promotion of Sciences.

\section{Appendix. Proof of Lemmas 2 and 3}

According to the proof of Theorem 2 in [23], we prove two lemmas.

\section{Proof of Lemma 2}

Substituting the second equation of 3.2 into the first one, we have

$$
\left\{\begin{array}{l}
\left(T^{\varepsilon}-\varepsilon^{2} \omega^{2}-\lambda\right) p_{m}=S^{\varepsilon}(\lambda) q_{m}, \quad z \in \mathbb{R}, \\
\left(M^{\varepsilon}-\omega^{2}-\lambda\right) q_{m}=-p_{m}, \\
p_{m}, q_{m} \in C_{\text {unif }}^{2}(\mathbb{R}),
\end{array}\right.
$$

where

$$
\begin{aligned}
T^{\varepsilon} \equiv & L^{\varepsilon}+\varepsilon k U^{\varepsilon} \chi_{0}^{\prime}\left(V^{\varepsilon}\right), \\
S^{\varepsilon}(\lambda) \equiv & \varepsilon k\left[\left\{-\varepsilon \theta(\varepsilon) U^{\varepsilon} \chi_{0}^{\prime}\left(V^{\varepsilon}\right)+U_{z}^{\varepsilon} \chi_{0}^{\prime}\left(V^{\varepsilon}\right)+2 U^{\varepsilon} \chi_{0}^{\prime \prime}\left(V^{\varepsilon}\right) V_{z}^{\varepsilon}\right\} \frac{\mathrm{d}}{\mathrm{d} z}\right. \\
& \left.+(\lambda+\gamma) U^{\varepsilon} \chi_{0}^{\prime}\left(V^{\varepsilon}\right)+\left\{U^{\varepsilon} \chi_{0}^{\prime \prime}\left(V^{\varepsilon}\right) V_{z}^{\varepsilon}\right\}_{z}\right] .
\end{aligned}
$$

Solving the first equation of A.1 with respect to $p_{m}$ and then using it, we rewrite the second equation of (A.1) as a scalar equation for $q_{m}$. To do it, we first consider the following eigenvalue problem for $T^{\varepsilon}$ :

$$
\left\{\begin{array}{l}
T^{\varepsilon} \phi=\zeta \phi \\
\phi( \pm \infty)=0
\end{array}\right.
$$

Let $\left\{\zeta_{n}^{\varepsilon}\right\}_{n \geqslant 0}$ and $\left\{\phi_{n}^{\varepsilon}\right\}_{n \geqslant 0}$ be, respectively, eigenvalues and the corresponding orthonormal eigenfunctions of the problem A.2. In order to study the properties of the principal eigenvalue $\zeta_{0}^{\varepsilon}$, it is convenient to introduce the stretched variable $\xi=z / \varepsilon$ into A.2. so that it is rewritten as

$$
\left\{\begin{array}{l}
\tilde{T}^{\varepsilon} \tilde{\phi}=\zeta \tilde{\phi} \\
\tilde{\phi}( \pm \infty)=0
\end{array}\right.
$$

where with $\left(\tilde{u}^{\varepsilon}, \tilde{v}^{\varepsilon}\right)(\xi)=\left(U^{\varepsilon}, V^{\varepsilon}\right)(\varepsilon \xi), \tilde{T}^{\varepsilon}$ is defined by

$$
\tilde{T}^{\varepsilon}=\frac{\mathrm{d}^{2}}{\mathrm{~d} \xi^{2}}+\left\{\theta(\varepsilon)-k \chi_{0}^{\prime}\left(\tilde{v}^{\varepsilon}\right) \tilde{v}_{z}^{\varepsilon}\right\} \frac{\mathrm{d}}{\mathrm{d} \xi}-\varepsilon k\left[\left\{\chi_{0}^{\prime}\left(\tilde{v}^{\varepsilon}\right) \tilde{v}_{z}^{\varepsilon}\right\}_{z}-\tilde{u}^{\varepsilon} \chi_{0}^{\prime}\left(\tilde{v}^{\varepsilon}\right)\right]+f^{\prime}\left(\tilde{u}^{\varepsilon}\right) .
$$


It is easy to show that the distribution of eigenvalues of the problem A.3 is the same as for A.2. Letting $\hat{\phi}_{n}^{\varepsilon}$ be the corresponding normalized eigenfunction, we conclude that

$$
\hat{\phi}_{n}^{\varepsilon}(\xi)=\sqrt{\varepsilon} \tilde{\phi}_{n}^{\varepsilon}(\xi)=\sqrt{\varepsilon} \phi_{n}^{\varepsilon}(\varepsilon \xi)
$$

Taking the limit $\varepsilon \downarrow 0$ in $\tilde{T}^{\varepsilon}$, we define $\tilde{T}^{*}$ by

$$
\tilde{T}^{*}=\frac{\mathrm{d}^{2}}{\mathrm{~d} \xi^{2}}+\left\{\theta^{*}+\frac{k}{2 \sqrt{\gamma}} \chi_{0}^{\prime}\left(\frac{1}{2 \gamma}\right)\right\} \frac{\mathrm{d}}{\mathrm{d} \xi}+f^{\prime}(W) .
$$

We thus find that $-(\mathrm{d} / \mathrm{d} \xi) W$ is a constant multiple of the positive normalized eigenfunction $\hat{\phi}_{0}^{*}$, which corresponds to the principal eigenvalue of $\tilde{T}^{*}$, and that

$$
-\frac{\mathrm{d}}{\mathrm{d} \xi} \tilde{u}^{\varepsilon} \rightarrow \rho^{*-1} \hat{\phi}_{0}^{*} \quad\left(=-\frac{\mathrm{d}}{\mathrm{d} \xi} W\right) \quad \text { as } \varepsilon \rightarrow 0,
$$

where $\rho^{*}=\|(\mathrm{d} / \mathrm{d} \xi) W\|_{L^{2}}^{-1}$.

Let $\zeta_{0}^{\varepsilon \sharp}$ be the principal eigenvalue and $\phi_{0}^{\varepsilon \sharp}$ be the corresponding eigenfunction of the adjoint operator of $T^{\varepsilon}$, say $T^{\varepsilon \sharp}$.

LEMMA 4 (Nishiura and Fujii [17]) We have

and

$$
\hat{\phi}_{0}^{\varepsilon} \rightarrow \hat{\phi}_{0}^{*} \quad \text { in } C_{\text {c.u. }}^{2}(\mathbb{R}) \text { as } \varepsilon \rightarrow 0
$$

$$
\hat{\phi}_{0}^{\varepsilon \sharp} \rightarrow \sigma^{*} e^{c^{*} \xi} \hat{\phi}_{0}^{*} \quad \text { in } C_{\text {c.u. }}^{2}(\mathbb{R}) \text { as } \varepsilon \rightarrow 0
$$

with $\sigma^{*}=\left\|e^{c^{*} \xi / 2} \hat{\phi}_{0}^{*}\right\|_{L^{2}}^{-2}$, where $\hat{\phi}_{0}^{\varepsilon \sharp}$ is defined similarly to $\hat{\phi}_{0}^{\varepsilon}$.

This lemma gives the asymptotic form of $\zeta_{0}^{\varepsilon}$ as $\varepsilon \downarrow 0$ as follows: Differentiating 2.1 with respect to $z$, we have

$$
\left\{\begin{aligned}
0= & \varepsilon^{2}\left(u_{z}\right)_{z z}+\varepsilon\left\{\theta(\varepsilon)-k \chi_{0}^{\prime}(v) v_{z}\right\}\left(u_{z}\right)_{z}-2 \varepsilon k\left\{\chi_{0}^{\prime \prime}(v) v_{z}^{2}+\chi_{0}^{\prime}(v) v_{z z}\right\} u_{z} \\
& -\varepsilon k\left\{\chi_{0}^{\prime \prime \prime}(v) v_{z}^{3}+3 \chi_{0}^{\prime \prime}(v) v_{z} v_{z z}+\chi_{0}^{\prime}(v) v_{z z z}\right\} u+f^{\prime}(u) u_{z}, \quad z \in \mathbb{R} \\
0= & v_{z z z}+\varepsilon \theta(\varepsilon) v_{z z}+u_{z}-\gamma v_{z} .
\end{aligned}\right.
$$

Substituting the second equation of A.5 into the first one, we obtain

$$
\begin{aligned}
0= & T^{\varepsilon} u_{z}-\varepsilon k\left\{\chi_{0}^{\prime \prime}(v) v_{z}^{2}+\chi_{0}^{\prime}(v) v_{z z}\right\} u_{z} \\
& -\varepsilon k\left[\chi_{0}^{\prime \prime \prime}(v) v_{z}^{3}+3 \chi_{0}^{\prime \prime}(v) v_{z} v_{z z}+\chi_{0}^{\prime}(v)\left\{-\varepsilon \theta(\varepsilon) v_{z z}+\gamma v_{z}\right\}\right] .
\end{aligned}
$$

By using the stretched variable $\xi=z / \varepsilon$, it follows from A.6 that the stretched travelling front solution $\left(\tilde{u}^{\varepsilon}, \tilde{v}^{\varepsilon}\right)(\xi)$ satisfies

$$
0=\tilde{T}^{\varepsilon}\left(\frac{1}{\varepsilon} \tilde{u}_{\xi}\right)-k\left\{\chi_{0}^{\prime \prime}(\tilde{v}) \tilde{v}_{z}^{2}+\chi_{0}^{\prime}(\tilde{v}) \tilde{v}_{z z}\right\} \tilde{u}_{\xi}+O(\varepsilon) .
$$

Multiplying A.7 by $\hat{\phi}_{0}^{\varepsilon \sharp}$ and integrating on $\mathbb{R}$, we have

$$
\left\langle\tilde{T}^{\varepsilon}\left(\frac{1}{\varepsilon} \tilde{u}_{\xi}\right), \hat{\phi}_{0}^{\varepsilon \sharp}\right\rangle=\left\langle k\left\{\chi_{0}^{\prime \prime}(\tilde{v}) \tilde{v}_{z}^{2}+\chi_{0}^{\prime}(\tilde{v}) \tilde{v}_{z z}\right\} \tilde{u}_{\xi}, \hat{\phi}_{0}^{\varepsilon \sharp}\right\rangle+O(\varepsilon),
$$


where $\langle\cdot, \cdot\rangle$ means the $L^{2}(\mathbb{R})$ inner product. Since

$$
\tilde{T}^{\varepsilon \sharp} \hat{\phi}_{0}^{\varepsilon \sharp}=\zeta_{0}^{\varepsilon} \hat{\phi}_{0}^{\varepsilon \sharp} \quad \text { and } \quad \tilde{v}_{z z}=-\varepsilon \theta(\varepsilon) \tilde{v}_{z}-\tilde{u}+\gamma \tilde{v},
$$

(A.8) can be rewritten as

$$
\frac{1}{\varepsilon} \zeta_{0}^{\varepsilon}\left\langle\tilde{u}_{\xi}, \hat{\phi}_{0}^{\varepsilon \sharp}\right\rangle=k\left\langle\left\{\chi_{0}^{\prime \prime}(\tilde{v}) \tilde{v}_{z}^{2}+\chi_{0}^{\prime}(\tilde{v})\left(-\varepsilon \theta(\varepsilon) \tilde{v}_{z}-\tilde{u}+\gamma \tilde{v}\right)\right\} \tilde{u}_{\xi}, \hat{\phi}_{0}^{\varepsilon \sharp}\right\rangle+O(\varepsilon) .
$$

By using Lemma 4 and A.9p, we know the following lemma:

LEMma 5 ([17]) For small $\varepsilon>0$,

$$
\hat{\zeta}_{0}^{*} \equiv \lim _{\varepsilon \downarrow 0} \frac{\zeta_{0}^{\varepsilon}}{\varepsilon}=k\left\{\frac{1}{4 \gamma} \chi_{0}^{\prime \prime}\left(\frac{1}{2 \gamma}\right)+\frac{1}{2} \chi_{0}^{\prime}\left(\frac{1}{2 \gamma}\right)-\frac{\chi_{0}^{\prime}\left(\frac{1}{2 \gamma}\right)\left\langle W_{\xi}, e^{c^{*} \xi} W_{\xi}\right\rangle_{\mathbb{R}_{-}}}{\left\langle W_{\xi}, e^{c^{*} \xi} W_{\xi}\right\rangle}\right\},
$$

where $\langle\cdot, \cdot\rangle_{\mathbb{R}_{-}}$means the $L^{2}\left(\mathbb{R}_{-}\right)$inner product with $\mathbb{R}_{-}=(-\infty, 0)$. Moreover, there is a positive constant $\mu$ independent of $\varepsilon$ such that other eigenvalues $\zeta_{n}^{\varepsilon}$ of $T^{\varepsilon}$ satisfy $\zeta_{n}^{\varepsilon}<-\mu$ for any integer $n>0$.

By using Lemma5, there is a constant $\nu>0$ depending on $\gamma$ and $\mu$ such that for any $\lambda \in \Lambda \equiv$ $\{\lambda \in \mathbb{C} \mid \operatorname{Re} \lambda>-\nu\}$, the first equation in $A .1$ ) can be rewritten as

$$
\begin{aligned}
p_{m} & =\left(T^{\varepsilon}-\varepsilon^{2} \omega^{2}-\lambda\right)^{-1} S^{\varepsilon}(\lambda) q_{m} \\
& \equiv \frac{\left\langle S^{\varepsilon}(\lambda) q_{m}, \phi_{0}^{\varepsilon \sharp}\right\rangle}{\zeta_{0}^{\varepsilon}-\varepsilon^{2} \omega^{2}-\lambda} \phi_{0}^{\varepsilon}+\left(T^{\varepsilon}-\varepsilon^{2} \omega^{2}-\lambda\right)^{\dagger} S^{\varepsilon}(\lambda) q_{m} .
\end{aligned}
$$

Here the first term means the projection on the space spanned by the eigenfunction $\phi_{0}^{\varepsilon}$ corresponding to the principal eigenvalue $\zeta_{0}^{\varepsilon}$ of $T^{\varepsilon}$ and $\left(T^{\varepsilon}-\varepsilon^{2} \omega^{2}-\lambda\right)^{\dagger}$ is the projection operator on its orthogonal complement in $L^{2}(\mathbb{R})$, respectively. We note that $\left(T^{\varepsilon}-\lambda\right)^{\dagger}$ is a uniformly $L^{2}$-bounded operator for $\lambda \in \Lambda$ and $\varepsilon>0$, and it satisfies

$$
\left\|\left(T^{\mathcal{E}}-\lambda\right)^{\dagger}\right\|_{L^{2} \rightarrow L^{2}} \leqslant \frac{M}{1+|\lambda|}
$$

where $M$ is a constant independent of $\varepsilon>0$ and $\lambda \in \Gamma$ (see [20, Lemma 1], for instance). Substituting A.11 into the second equation of A.1, we obtain the equation for $q_{m}$ as follows:

$$
\begin{aligned}
\lambda q_{m}= & \frac{\left\langle S^{\varepsilon}(\lambda) q_{m}, \phi_{0}^{\varepsilon \sharp}\right\rangle}{\zeta_{0}^{\varepsilon}-\varepsilon^{2} \omega^{2}-\lambda} \phi_{0}^{\varepsilon}+\left(T^{\varepsilon}-\varepsilon^{2} \omega^{2}-\lambda\right)^{\dagger} S^{\varepsilon}(\lambda) q_{m} \\
& +\left(q_{m}\right)_{z z}+\varepsilon \theta(\varepsilon)\left(q_{m}\right)_{z}-\left(\gamma+\omega^{2}\right) q_{m} .
\end{aligned}
$$

Therefore, A.13 is solved by using the following bilinear form:

$$
\begin{aligned}
0= & -\left\langle\left(q_{m}\right)_{z}, \psi_{z}\right\rangle+\varepsilon \theta(\varepsilon)\left\langle\left(q_{m}\right)_{z}, \psi\right\rangle-\left(\gamma+\omega^{2}+\lambda\right)\left\langle q_{m}, \psi\right\rangle \\
& +\frac{\left\langle S^{\varepsilon}(\lambda) q_{m}, \phi_{0}^{\varepsilon \sharp}\right\rangle}{\zeta_{0}^{\varepsilon}-\varepsilon^{2} \omega^{2}-\lambda}\left\langle\phi_{0}^{\varepsilon}, \psi\right\rangle+\left\langle\left(T^{\varepsilon}-\varepsilon^{2} \omega^{2}-\lambda\right)^{\dagger} S^{\varepsilon}(\lambda) q_{m}, \psi\right\rangle
\end{aligned}
$$

for $q_{m}, \psi \in H^{1}(\mathbb{R})$, where $q_{m}$ is normalized as $\left\|q_{m}\right\|_{L^{2}}=1$. 
LEMMA 6 There is a positive constant $M_{c}$ independent of $0<\varepsilon<1$ and $\omega>0$ such that any eigenvalue $\lambda \in \Lambda_{\delta} \equiv\left\{\lambda \in \Lambda|| \varepsilon^{2} \omega^{2}+\lambda \mid \geqslant \delta\right\}$ of (A.1) satisfies $\left|\varepsilon^{2} \omega^{2}+\lambda\right|<M_{c}$ for any given $\delta>0$.

Proof. Setting $\psi=q_{m}$ in (A.14), we have

$$
\begin{aligned}
\left(\gamma+\omega^{2}+\lambda\right)\left\|q_{m}\right\|_{L^{2}}^{2}+\left\|q_{m z}\right\|_{L^{2}}^{2}= & \frac{\left\langle S^{\varepsilon}(\lambda) q_{m}, \phi_{0}^{\varepsilon \sharp}\right\rangle}{\zeta_{0}^{\varepsilon}-\varepsilon^{2} \omega^{2}-\lambda}\left\langle\phi_{0}^{\varepsilon}, q_{m}\right\rangle \\
& +\left\langle\left(T^{\varepsilon}-\varepsilon^{2} \omega^{2}-\lambda\right)^{\dagger} S^{\varepsilon}(\lambda) q_{m}, q_{m}\right\rangle .
\end{aligned}
$$

For the real part of (A.15), it follows from (A.12) that

$$
\begin{aligned}
\left(\gamma+\omega^{2}+\operatorname{Re} \lambda\right)\left\|q_{m}\right\|_{L^{2}}^{2}+ & \left\|\left(q_{m}\right)_{z}\right\|_{L^{2}}^{2} \\
= & \operatorname{Re}\left\{\frac{\left\langle S^{\varepsilon}(\lambda) q_{m}, \phi_{0}^{\varepsilon \sharp}\right\rangle}{\zeta_{0}^{\varepsilon}-\varepsilon^{2} \omega^{2}-\lambda}\left\langle\phi_{0}^{\varepsilon}, q_{m}\right\rangle+\left\langle\left(T^{\varepsilon}-\varepsilon^{2} \omega^{2}-\lambda\right)^{\dagger} S^{\varepsilon}(\lambda) q_{m}, q_{m}\right\rangle\right\} \\
\leqslant & \frac{\left|\left\langle S^{\varepsilon}(\lambda) q_{m}, \phi_{0}^{\varepsilon \sharp}\right\rangle\right|}{\left|\zeta_{0}^{\varepsilon}-\varepsilon^{2} \omega^{2}-\lambda\right|}\left|\left\langle\phi_{0}^{\varepsilon}, q_{m}\right\rangle\right|+\left|\left\langle\left(T^{\varepsilon}-\varepsilon^{2} \omega^{2}-\lambda\right)^{\dagger} S^{\varepsilon}(\lambda) q_{m}, q_{m}\right\rangle\right| \\
\leqslant & C\left\|q_{m}\right\|_{L^{2}}\left\{\left\|\left(q_{m}\right)_{z}\right\|_{L^{2}}+\varepsilon\left(\gamma+\omega^{2}+|\lambda|\right)\left\|q_{m}\right\|_{L^{2}}+\left\|q_{m}\right\|_{L^{2}}\right\} \\
& \times\left\{\frac{1}{\left|\zeta_{0}^{\varepsilon}-\varepsilon^{2} \omega^{2}-\lambda\right|}+\frac{M}{1+\left|\varepsilon^{2} \omega^{2}+\lambda\right|}\right\},
\end{aligned}
$$

where $C$ is a positive constant independent of $0<\varepsilon<1$ and $\omega>0$. Since $\left\|q_{m}\right\|_{L^{2}}\left\|\left(q_{m}\right)_{z}\right\|_{L^{2}} \leqslant$ $\frac{1}{4 C_{1}}\left\|q_{m}\right\|_{L^{2}}^{2}+C_{1}\left\|\left(q_{m}\right)_{z}\right\|_{L^{2}}^{2}$ for any $C_{1}>0$, it follows from the above inequality and $\left\|q_{m}\right\|_{L^{2}}=1$ that

$$
\begin{aligned}
\gamma+\omega^{2} & +\operatorname{Re} \lambda+\left[1-C C_{1}\left\{\frac{1}{\left|\zeta_{0}^{\varepsilon}-\varepsilon^{2} \omega^{2}-\lambda\right|}+\frac{M}{1+\left|\varepsilon^{2} \omega^{2}+\lambda\right|}\right\}\right]\left\|\left(q_{m}\right)_{z}\right\|_{L^{2}}^{2} \\
& <C\left(\frac{1}{4 C_{1}}+\varepsilon\left(\gamma+\omega^{2}+|\lambda|\right)+1\right)\left\{\frac{1}{\left|\zeta_{0}^{\varepsilon}-\varepsilon^{2} \omega^{2}-\lambda\right|}+\frac{M}{1+\left|\varepsilon^{2} \omega^{2}+\lambda\right|}\right\}
\end{aligned}
$$

where $C_{1}$ is a small positive constant such that

$$
1-C C_{1}\left\{\frac{1}{\left|\zeta_{0}^{\varepsilon}-\varepsilon^{2} \omega^{2}-\lambda\right|}+\frac{M}{1+\left|\varepsilon^{2} \omega^{2}+\lambda\right|}\right\}>0 .
$$

Therefore, the left hand side of A.16 is larger than the right one as $\left|\varepsilon^{2} \omega^{2}+\operatorname{Re} \lambda\right| \rightarrow \infty$. This is a contradiction so that $\left|\varepsilon^{2} \omega^{2}+\operatorname{Re} \lambda\right|$ is finite.

On the other hand, it follows from (A.15) that

$$
\operatorname{Im} \lambda\left\|q_{m}\right\|_{L^{2}}^{2}=\operatorname{Im}\left\{\frac{\left\langle S^{\varepsilon}(\lambda) q_{m}, \phi_{0}^{\varepsilon \sharp}\right\rangle}{\zeta_{0}^{\varepsilon}-\varepsilon^{2} \omega^{2}-\lambda}\left\langle\phi_{0}^{\varepsilon}, q_{m}\right\rangle+\left\langle\left(T^{\varepsilon}-\varepsilon^{2} \omega^{2}-\lambda\right)^{\dagger} S^{\varepsilon}(\lambda) q_{m}, q_{m}\right\rangle\right\} .
$$

By using the same argument as above, one shows that $\left\|\left(q_{m}\right)_{z}\right\|_{L^{2}} \rightarrow \infty$ as $\operatorname{Im} \lambda \rightarrow \infty$. Noting that A.16 is valid in this case, one finds that as $|\operatorname{Im} \lambda| \rightarrow \infty$, the left hand side becomes larger than the right one for small $\varepsilon>0$, which is a contradiction. Hence, $|\operatorname{Im} \lambda|<\infty$, that is, there is a positive constant $M_{c}$ such that $\left|\varepsilon^{2} \omega^{2}+\lambda\right|<M_{c}$ for any eigenvalue $\lambda \in \Lambda_{\delta}$. 
LEMma 7 If $q_{m}$ is the eigenfunction corresponding to any eigenvalue $\lambda \in \Lambda_{\delta}$ satisfying $\left|\varepsilon^{2} \omega^{2}+\lambda\right|<M_{c}$ of (A.1), then there is a positive constant $\hat{C}$ such that

$$
1 \leqslant\left\|q_{m}\right\|_{H^{2}} \leqslant \hat{C} .
$$

Proof. From A.15, there is a positive constant $C_{3}$ satisfying

$$
\begin{aligned}
\left\|\left(q_{m}\right)_{z}\right\|_{L^{2}}^{2} \leqslant & \gamma+\omega^{2}+|\lambda|+\left|\frac{\left\langle S^{\varepsilon}(\lambda) q_{m}, \phi_{0}^{\varepsilon \sharp}\right\rangle}{\zeta_{0}^{\varepsilon}-\varepsilon^{2} \omega^{2}-\lambda}\left\langle\phi_{0}^{\varepsilon}, q_{m}\right\rangle\right|+\left|\left\langle\left(T^{\varepsilon}-\varepsilon^{2} \omega^{2}-\lambda\right)^{\dagger} S^{\varepsilon}(\lambda) q_{m}, q_{m}\right\rangle\right| \\
\leqslant & \gamma+\omega^{2}+|\lambda|+C_{3}\left\{\left\|\left(q_{m}\right)_{z}\right\|_{L^{2}}+\varepsilon\left(\gamma+\omega^{2}+|\lambda|\right)+1\right\} \\
& \times\left\{\frac{1}{\left|\zeta_{0}^{\varepsilon}-\varepsilon^{2} \omega^{2}-\lambda\right|}+\frac{M}{1+\left|\varepsilon^{2} \omega^{2}+\lambda\right|}\right\} .
\end{aligned}
$$

By using $\left\|q_{m}\right\|_{L^{2}}=1$ and the argument similar to A.16, we have

$$
\begin{aligned}
& {\left[1-\frac{C_{3} C_{4}}{2}\left\{\frac{1}{\left|\zeta_{0}^{\varepsilon}-\varepsilon^{2} \omega^{2}-\lambda\right|}+\frac{M}{1+\left|\varepsilon^{2} \omega^{2}+\lambda\right|}\right\}\right]\left\|\left(q_{m}\right)_{z}\right\|_{L^{2}}^{2}} \\
& \leqslant \gamma+\omega^{2}+|\lambda|+C_{3}\left\{\frac{1}{2 C_{4}}+\varepsilon\left(\gamma+\omega^{2}+|\lambda|\right)+1\right\}\left\{\frac{1}{\left|\zeta_{0}^{\varepsilon}-\varepsilon^{2} \omega^{2}-\lambda\right|}+\frac{M}{1+\left|\varepsilon^{2} \omega^{2}+\lambda\right|}\right\}
\end{aligned}
$$

for a small positive constant $C_{4}$. Thus, there is a positive constant $C_{5}$ satisfying $\left\|\left(q_{m}\right)_{z}\right\|_{L^{2}} \leqslant C_{5}$. Moreover, it follows from A.13 that $\left\|\left(q_{m}\right)_{z z}\right\|_{L^{2}} \leqslant C_{6}\left\|q_{m}\right\|_{H^{1}}$ with some positive constant $C_{6}$. Therefore, there is a positive constant $\hat{C}$ satisfying $1 \leqslant\left\|q_{m}\right\|_{H^{2}} \leqslant \hat{C}$.

To show the distribution of eigenvalues of (A.1) in $\left\{\lambda \in \Lambda_{\delta}|| \varepsilon^{2} \omega^{2}+\lambda \mid<M_{c}\right\}$, we need the following two lemmas:

LEMMA 8 ([20]) Let $F(u, v)$ be a smooth function of $u$ and $v$. Then, for any $s \in(0,1 / 2)$,

$$
\lim _{\varepsilon \rightarrow 0}\left\|\left(T^{\varepsilon}-\lambda\right)^{\dagger}\left(F^{\varepsilon} \cdot\right)-\frac{F^{0}}{f^{\prime}(W)-\lambda}\right\|_{\mathcal{L}\left(L^{2}, H^{-s}\right)}=0 \quad \text { uniformly in } \lambda \in \Lambda,
$$

where $\left(u^{0}, v^{0}\right)(z)$ is given in Theorem $1, F^{\varepsilon}=F\left(U^{\varepsilon}(z), V^{\varepsilon}(z)\right)$ and $F^{0}=F\left(u^{0}(z), v^{0}(z)\right)$.

Lemma 9 There is $\varepsilon_{0}>0$ such that no $\lambda \in \Lambda_{\delta}$ satisfying $\left|\varepsilon^{2} \omega^{2}+\lambda\right|<M_{c}$ is an eigenvalue of (A.1) for any $0<\varepsilon<\varepsilon_{0}$.

Proof. Let $\lambda$ be an eigenvalue of (A.1). By Lemma 7 , there are positive constants $C_{1}, C_{2}, C_{3}$ and $C_{4}$ independent of $\varepsilon$ and $\mu$ such that

$$
\left|\left\langle\phi_{0}^{\varepsilon}, q_{m}\right\rangle\right| \leqslant\left\|q_{m}\right\|_{L^{\infty}} C_{1} \sqrt{\varepsilon} \leqslant C_{2} \sqrt{\varepsilon}
$$

and

$$
\left|\left\langle S^{\varepsilon}(\lambda) q_{m}, \phi_{0}^{\varepsilon \sharp}\right\rangle\right| \leqslant\left\|S^{\varepsilon}(\lambda) q_{m}\right\|_{L^{2}}\left\|\phi_{0}^{\varepsilon \sharp}\right\|_{L^{2}} \leqslant C_{3}\left\|S^{\varepsilon}(\lambda) q_{m}\right\|_{L^{2}}<C_{4}(1+\varepsilon|\lambda|)
$$

for small $\varepsilon>0$. Moreover, it follows from (A12) that for any $\lambda \in \Lambda$,

$$
\left(T^{\varepsilon}-\lambda\right)^{\dagger}\left(\varepsilon U_{z}^{\varepsilon} \cdot\right) \rightarrow 0 \quad \text { uniformly on any bounded set in } L^{2}(\mathbb{R}) \text { as } \varepsilon \downarrow 0 .
$$

Therefore, Lemmas 7 and 8 show that the left hand side of $(A .15)$ is larger than the right one for small $\varepsilon>0$, which is a contradiction.

We thus find that there is no eigenvalue of (A.1) in $\Lambda_{\delta}=\left\{\lambda \in \Lambda|| \varepsilon^{2} \omega^{2}+\lambda \mid>\delta\right\}$. 
Proof of Lemma 3

We now show the asymptotic form of the eigenvalue $\lambda$ satisfying (3.4) for sufficiently small $\varepsilon>0$ by using A.14 and Lemmas 4 and 5. From A.14 we define the following bilinear form:

$$
B^{\omega, \lambda, \varepsilon}(p, q)=\left\langle p_{z}, q_{z}\right\rangle-\varepsilon \theta(\varepsilon)\left\langle p_{z}, q\right\rangle+\left(\gamma+\omega^{2}+\lambda\right)\langle p, q\rangle
$$

for $p, q \in H^{1}(\mathbb{R})$. It is easy to see that there are two positive constants $C_{3}$ and $C_{4}$ such that

$$
\left|B^{\omega, \lambda, \varepsilon}(p, q)\right| \leqslant C_{3}\|p\|_{H^{1}}\|q\|_{H^{1}}
$$

and

$$
\left|B^{\omega, \lambda, \varepsilon}(q, q)\right| \geqslant C_{4}\|q\|_{H^{1}}^{2}
$$

for $\lambda$ and $\omega$ satisfying 3.4. By the Lax-Milgram theorem, for any $h \in H^{-1}(\mathbb{R})$ there exists $q \in$ $H^{1}(\mathbb{R})$ such that

$$
B^{\omega, \lambda, \varepsilon}(q, \psi)=\langle h, \psi\rangle \quad \text { for any } \psi \in H^{1}(\mathbb{R}) .
$$

Then a bounded linear operator $K^{\omega, \lambda, \varepsilon}$ from $H^{-1}(\mathbb{R})$ to $H^{1}(\mathbb{R})$ can be defined by $q=K^{\omega, \lambda, \varepsilon} h$. It follows from (3.4) that

$$
B^{\omega, \lambda, \varepsilon}\left(q_{m}, \psi\right)=\frac{\left\langle S^{\varepsilon}(\lambda) q_{m}, \phi_{0}^{\varepsilon \sharp}\right\rangle}{\zeta_{0}^{\varepsilon}-\varepsilon^{2} \omega^{2}-\lambda}\left\langle\phi_{0}^{\varepsilon}, \psi\right\rangle+\left\langle\left(T^{\varepsilon}-\varepsilon^{2} \omega^{2}-\lambda\right)^{\dagger} S^{\varepsilon}(\lambda) q_{m}, \psi\right\rangle
$$

for any $\psi \in H^{1}(\mathbb{R})$. Therefore, A.18 is rewritten as

$$
\begin{aligned}
q_{m} & =\frac{\left\langle S^{\varepsilon}(\lambda) q_{m}, \phi_{0}^{\varepsilon \sharp}\right\rangle}{\zeta_{0}^{\varepsilon}-\varepsilon^{2} \omega^{2}-\lambda} K^{\omega, \lambda, \varepsilon}\left(\phi_{0}^{\varepsilon}\right)+K^{\omega, \lambda, \varepsilon}\left(\left(T^{\varepsilon}-\varepsilon^{2} \omega^{2}-\lambda\right)^{\dagger} S^{\varepsilon}(\lambda) q_{m}\right) \\
& =\frac{\left\langle S^{\varepsilon}(\lambda) q_{m}, \phi_{0}^{\varepsilon \sharp} / \sqrt{\varepsilon}\right\rangle}{\left(\zeta_{0}^{\varepsilon}-\varepsilon^{2} \omega^{2}-\lambda\right) / \varepsilon} K^{\omega, \lambda, \varepsilon}\left(\phi_{0}^{\varepsilon} / \sqrt{\varepsilon}\right)+K^{\omega, \lambda, \varepsilon}\left(\left(T^{\varepsilon}-\varepsilon^{2} \omega^{2}-\lambda\right)^{\dagger} S^{\varepsilon}(\lambda) q_{m}\right) .
\end{aligned}
$$

Putting

$$
q_{m}=\alpha K^{\omega, \lambda, \varepsilon}\left(\phi_{0}^{\varepsilon} / \sqrt{\varepsilon}\right)+K^{\omega, \lambda, \varepsilon}(b)
$$

with $\left\langle b, \phi_{0}^{\varepsilon \sharp}\right\rangle=0$, we have

$$
\left\{\begin{array}{l}
\frac{\alpha\left(\zeta_{0}^{\varepsilon}-\varepsilon^{2} \omega^{2}-\lambda\right)}{\varepsilon}=\alpha\left\langle S^{\varepsilon}(\lambda) K^{\omega, \lambda, \varepsilon}\left(\phi_{0}^{\varepsilon} / \sqrt{\varepsilon}\right), \phi_{0}^{\varepsilon \sharp} / \sqrt{\varepsilon}\right\rangle+\left\langle S^{\varepsilon}(\lambda) K^{\omega, \lambda, \varepsilon}(b), \phi_{0}^{\varepsilon \sharp} / \sqrt{\varepsilon}\right\rangle, \\
b=\left(T^{\varepsilon}-\varepsilon^{2} \omega^{2}-\lambda\right)^{\dagger} S^{\varepsilon}(\lambda)\left(\alpha K^{\omega, \lambda, \varepsilon}\left(\phi_{0}^{\varepsilon} / \sqrt{\varepsilon}\right)+K^{\omega, \lambda, \varepsilon}(b)\right) .
\end{array}\right.
$$

Then the second equation of A.20 can be rewritten as

$$
\left[I-\left(T^{\varepsilon}-\varepsilon^{2} \omega^{2}-\lambda\right)^{\dagger} S^{\varepsilon}(\lambda) K^{\omega, \lambda, \varepsilon}\right] b=\alpha\left(T^{\varepsilon}-\varepsilon^{2} \omega^{2}-\lambda\right)^{\dagger} S^{\varepsilon}(\lambda) K^{\omega, \lambda, \varepsilon}\left(\phi_{0}^{\varepsilon} / \sqrt{\varepsilon}\right) .
$$

We remark that $K^{\omega, \lambda, \varepsilon}$ is also a bounded operator from $L^{2}(\mathbb{R})$ to $H^{2}(\mathbb{R})$. It follows that $K^{\omega, \lambda, \varepsilon}\left(\phi_{0}^{\varepsilon} / \sqrt{\varepsilon}\right) \in H^{2}(\mathbb{R})$, since $\phi_{0}^{\varepsilon} / \sqrt{\varepsilon} \in L^{2}(\mathbb{R})$ for small $\varepsilon>0$. Moreover,

$$
\left\|S^{\varepsilon}(\lambda) K^{\omega, \lambda, \varepsilon}\left(\phi_{0}^{\varepsilon} / \sqrt{\varepsilon}\right)\right\|_{L^{2}}=O(\varepsilon)
$$


and

$$
\left\|\left(T^{\varepsilon}-\varepsilon^{2} \omega^{2}-\lambda\right)^{\dagger} S^{\varepsilon}(\lambda) K^{\omega, \lambda, \varepsilon}\right\|_{L^{2} \rightarrow L^{2}}=O(\varepsilon) \quad \text { for small } \varepsilon>0 .
$$

Therefore, the operator $I-\left(T^{\varepsilon}-\varepsilon^{2} \omega^{2}-\lambda\right)^{\dagger} S^{\varepsilon}(\lambda) K^{\omega, \lambda, \varepsilon}$ has a bounded inverse from $L^{2}(\mathbb{R})$ to $L^{2}(\mathbb{R})$. We have $b=\left[I-\left(T^{\varepsilon}-\varepsilon^{2} \omega^{2}-\lambda\right)^{\dagger} S^{\varepsilon}(\lambda) K^{\omega, \lambda, \varepsilon}\right]^{-1} \alpha S^{\varepsilon}(\lambda) K^{\omega, \lambda, \varepsilon}\left(\phi_{0}^{\varepsilon} / \sqrt{\varepsilon}\right)=O(\varepsilon)$ in $L^{2}(\mathbb{R})$, which implies that $K^{\omega, \lambda, \varepsilon}(b)=O(\varepsilon)$ in $H^{2}(\mathbb{R})$ for small $\varepsilon>0$. If $\alpha=0$, we know $b=0$, that is, $\left(p_{m}, q_{m}\right)=(0,0)$. This contradicts the fact that $\left(p_{m}, q_{m}\right)$ is an eigenfunction, so that $\alpha \neq 0$.

Next, we consider the limiting form of the first equation in A.20, taking $\varepsilon \downarrow 0$.

LEMma 10 ([17]) The following relations for $\phi_{0}^{\varepsilon}$ and $\phi_{0}^{\varepsilon \sharp}$ hold:

$$
\begin{aligned}
& \lim _{\varepsilon \downarrow 0} \frac{\phi_{0}^{\varepsilon}}{\sqrt{\varepsilon}}=\rho^{*} \delta_{0} \quad \text { in } H^{-1}(\mathbb{R}) ; \\
& \lim _{\varepsilon \downarrow 0} \frac{\phi_{0}^{\varepsilon \sharp}}{\sqrt{\varepsilon}}=\rho^{*} \sigma^{*} \delta_{0} \quad \text { in } H^{-1}(\mathbb{R}) ; \\
& \lim _{\varepsilon \downarrow 0} \sqrt{\varepsilon} U_{z}^{\varepsilon} \chi_{0}^{\prime}\left(V^{\varepsilon}\right) \phi_{0}^{\varepsilon \sharp}=-\rho^{*} \sigma^{*} \chi_{0}^{\prime}\left(\frac{1}{2 \gamma}\right)\left\langle W_{\xi}, e^{c \xi} W_{\xi}\right\rangle \delta_{0} \quad \text { in } H^{-1}(\mathbb{R}) ; \\
& \lim _{\varepsilon \downarrow 0} \sqrt{\varepsilon} U_{z}^{\varepsilon} \chi_{0}^{\prime \prime}\left(V^{\varepsilon}\right) V_{z}^{\varepsilon} \phi_{0}^{\varepsilon \sharp}=\frac{\rho^{*} \sigma^{*}}{2 \sqrt{\gamma}} \chi_{0}^{\prime \prime}\left(\frac{1}{2 \gamma}\right)\left\langle W_{\xi}, e^{c \xi} W_{\xi}\right\rangle \delta_{0} \quad \text { in } H^{-1}(\mathbb{R}),
\end{aligned}
$$

where $\delta_{0}$ is the Dirac $\delta$-function at $z=0$.

If $\omega$ is large to be of the order $O\left(\varepsilon^{-1 / 2}\right)$ for small $\varepsilon>0$, Lemma 10 leads to

$$
\alpha\left(\hat{\zeta}_{0}^{*}-\varepsilon \omega^{2}-\tau^{*}\right)=\alpha \lim _{\varepsilon \downarrow 0}\left\langle S^{\varepsilon}(\lambda) K^{\omega, \lambda, \varepsilon}\left(\phi_{0}^{\varepsilon} / \sqrt{\varepsilon}\right), \phi_{0}^{\varepsilon \sharp} / \sqrt{\varepsilon}\right\rangle
$$

for $\lambda=\varepsilon \tau(\varepsilon)$ with $\lim _{\varepsilon \downarrow 0} \tau(\varepsilon)=\tau^{*}$. Putting $\hat{q}=\alpha K^{\omega, \lambda, \varepsilon}\left(\phi_{0}^{\varepsilon} / \sqrt{\varepsilon}\right)$, we have the following equation:

$$
\left\langle\hat{q}_{z}, \psi_{z}\right\rangle-\varepsilon \theta(\varepsilon)\left\langle\hat{q}_{z}, \psi\right\rangle+\left(\gamma+\omega^{2}+\lambda\right)\langle\hat{q}, \psi\rangle=\alpha\left\langle\phi_{0}^{\varepsilon} / \sqrt{\varepsilon}, \psi\right\rangle \quad \text { for any } \psi \in H^{1}(\mathbb{R}) .
$$

By the above equation and Lemma 10 , we can see that $\hat{q}^{*}=\lim _{\varepsilon \downarrow 0} \hat{q}$ with $\left\|\hat{q}^{*}\right\|_{L^{2}}=1$ satisfies

$$
\left\{\begin{array}{l}
\hat{q}_{z z}^{*}-\left(\gamma+\omega^{2}\right) \hat{q}^{*}=0, \quad z \in(-\infty, 0) \cup(0,+\infty) \\
\hat{q}^{*}( \pm \infty)=0, \quad \hat{q}^{*}(0)=\psi
\end{array}\right.
$$

and

$$
\hat{q}_{z}^{*}(-0)-\hat{q}_{z}^{*}(+0)=\alpha \rho^{*},
$$

where $\psi$ is some constant. The solution $\hat{q}^{*}$ of A.22 is easily seen to be

$$
\hat{q}^{*}(z)= \begin{cases}\psi \exp \left(-\sqrt{\gamma+\omega^{2}} z\right) & (z>0), \\ \psi \exp \left(\sqrt{\gamma+\omega^{2}} z\right) & (z>0),\end{cases}
$$

so that A.23 gives

$$
\alpha \rho^{*}=2 \psi \sqrt{\gamma+\omega^{2}} .
$$


By using the first equation of A.20, Lemma 6 and A.24, we find that

$$
\begin{aligned}
\alpha\left(\hat{\zeta}_{0}^{*}-\varepsilon \omega^{2}-\tau^{*}\right)= & -k \rho^{*} \sigma^{*} \chi_{0}^{\prime}\left(\frac{1}{2 \gamma}\right)\left[2 \psi \sqrt{\gamma+\omega^{2}}\left\langle W_{\xi}, e^{c \xi} W_{\xi}\right\rangle_{\mathbb{R}_{-}}\right. \\
& \left.-\psi \sqrt{\gamma+\omega^{2}}\left\langle W_{\xi}, e^{c \xi} W_{\xi}\right\rangle\right]+\frac{k \rho^{*} \sigma^{*} \chi_{0}^{\prime \prime}(1 / 2 \gamma)}{2 \sqrt{\gamma}} \psi\left\langle W_{\xi}, e^{c \xi} W_{\xi}\right\rangle .
\end{aligned}
$$

By the definitions of $\rho^{*}, \sigma^{*}$ and Lemma 4 , we have

$$
\left\langle W_{\xi}, e^{c \xi} W_{\xi}\right\rangle=\left\|e^{c \xi / 2}\left(-\rho^{*^{-1}} \hat{\phi}_{0}^{*}\right)\right\|_{L^{2}}^{2}=\left(\rho^{*}\right)^{-2}\left(\sigma^{*}\right)^{-1}
$$

It follows from A.25, A.26 and A.27 that

$$
\begin{aligned}
\hat{\zeta}_{0}^{*}-\varepsilon \omega^{2}-\tau^{*}= & -k \rho^{*^{2}} \sigma^{*} \chi_{0}^{\prime}\left(\frac{1}{2 \gamma}\right)\left\langle W_{\xi}, e^{c \xi} W_{\xi}\right\rangle_{\mathbb{R}_{-}} \\
& +\frac{k}{2} \chi_{0}^{\prime}\left(\frac{1}{2 \gamma}\right)+\frac{k}{4 \sqrt{\gamma} \sqrt{\gamma+\omega^{2}}} \chi_{0}^{\prime \prime}\left(\frac{1}{2 \gamma}\right) .
\end{aligned}
$$

Therefore, by using A.10 and A.27, A.28 can be rewritten as follows:

$$
\tau^{*}=-\varepsilon \omega^{2}+\frac{k}{4 \sqrt{\gamma}}\left(\frac{1}{\sqrt{\gamma}}-\frac{1}{\sqrt{\gamma+\omega^{2}}}\right) \chi_{0}^{\prime \prime}\left(\frac{1}{2 \gamma}\right) .
$$

On the other hand, in the case $\lim _{\mathcal{L} \downarrow 0} \varepsilon \omega^{2}=\infty$, the above argument shows $\lim _{\varepsilon \downarrow 0} \tau=-\infty$. Therefore, putting $\omega=m \pi / \ell$ and $\tau_{m}=\tau^{*}$, we can complete the proof of Lemma 3 by using the implicit function theorem.

\section{REFERENCES}

1. Aronson, D. G., \& Weinberger, H. F. Nonlinear diffusion in population genetics, combustion and nerve propagation. Partial Differential Equations and Related Topics, J. A. Goldstein (ed.), Lecture Notes in Math. 446, Springer, New York (1975), 5-49. Zbl 0325.35050 MR 0427837

2. Aronson, D. G., \& Weinberger, H. F. Multidimensional nonlinear diffusion arising in population genetics. Adv. Math. 30 (1987), 33-76. Zbl 0407.92014 MR 0511740

3. Brenner, M. P., Levitov, L. S., \& Budrene, E. O. Physical mechanisms for chemotactic pattern formation by bacteria. Biophys. J. 74 (1998), 1677-1693.

4. Budrene, E. O., \& Berg, H. C. Complex patterns formed by motile cells of Escherichia coli. Nature 349 (1991), 630-633.

5. Budrene, E. O., \& Berg, H. C. Dynamics of formation of symmetrical patterns by chemotactic bacteria. Nature 376 (1995), 49-53.

6. Ezoe, H., IwASA, Y., \& UMEDA, T. Aggregation by chemotactic random walk: Drifting clusters and chemotactic friction. J. Theor. Biol. 168 (1994), 256-267.

7. FIFE, P. C. Boundary and interior transition layer phenomena for pairs of second-order differential equations. J. Math. Anal. Appl. 54 (1976), 497-521. Zbl 0345.34044 MR 0419961

8. FifE, P. C., \& MCLEOD, J. B. The approach of solutions of nonlinear diffusion equation to travelling wave solutions. Arch. Ration. Mech. Anal. 65 (1977), 335-361. Zbl 0361.35035 MR 0442480 
9. Ford, R. M., \& Lauffenburger, D. A. Analysis of chemotactic bacterial distributions in population migration essays using a mathematical model applicable to steep or shallow attractant gradients. Bull. Math. Biol. 53 (1991), 721-749. Zbl 0729.92029

10. Jones, C. K. R. T. Spherically symmetric solutions of a reaction-diffusion equation. J. Differential Equations 49 (1983), 142-169. Zbl 0523.35059 MR 0704268

11. Jones, C. K. R. T. Asymptotic behavior of a reaction-diffusion equation in higher space dimensions. Rocky Mountain J. Math. 13 (1983), 355-364. Zbl 0528.35054 MR 0702830

12. KAWASAKI, K., \& SHIGESADA, N. Modeling pattern formation of chemotactic bacteria. Manuscript.

13. Mimura, M., TABata, M., \& Hosono, Y. Multiple solutions of two-point boundary value problems of Neumann type with a small parameter. SIAM J. Math. Anal. 11 (1980), 613-631. Zbl 0438.34014 MR 0579554

14. Mimura, M., \& Tsujikawa, T. Aggregating pattern dynamics in a chemotaxis model including growth. Phys. A 230 (1996), 499-543.

15. De Mottoni, P., \& Schatzman, M. Geometrical evolution of developed interfaces. Trans. Amer. Math. Soc. 347 (1995), 1533-1589. Zbl 0840.35010 MR 1672406

16. Murray, J. D. Mathematical Biology. Springer, Berlin (1989). Zbl 0682.92001 MR 1007836

17. NishiurA, Y., \& FUJII, H. Stability of singularly perturbed solutions to systems of reaction-diffusion equations. SIAM J. Math. Anal. 18 (1987), 1726-1770. Zbl 0638.35010 MR 0911661

18. SchaAf, R. Stationary solutions of chemotaxis system. Trans. Amer. Math. Soc. 292 (1985), 531-556. Zbl 0637.35007 MR 0808736

19. STEVENS, A. A stochastic cellular automaton modeling gliding and aggregation of myxobacteria. SIAM J. Appl. Math. 61 (2000), 172-182. Zbl 0992.92005 MR 1776392

20. TANiguChi, M. A uniform convergence theorem for singular limit eigenvalue problems. Adv. Differential Equations 8 (2003), 29-54. Zbl 1030.35103 MR 1946557

21. TANiguchi, M. Instability of planar traveling waves in bistable reaction-diffusion systems. Discrete Contin. Dyn. Syst. Ser. B 3 (2003), 21-44. ZZ1 1024.35047| MR 1951566

22. Tsimring, L., Levine, H., Aronson, I., Ben-Jacob, E., Cohen, I., Shochet, O., \& Reynolds, W. N. Aggregation patterns in stressed bacteria. Phys. Rev. Lett. 75 (1995), 1859-1862.

23. TsujIKAWA, T. Singular limit analysis of planar equilibrium solutions to a chemotaxis model equation with growth. Methods Appl. Anal. 3 (1996), 401-431. Zbl 0892.35084 MR 1437787

24. Volpert, A., Volpert, Vit. A., \& Volpert, Vl. A. Traveling Wave Solutions of Parabolic Systems. AMS, Providence, RI (1994). Zbl 1001.35060 MR 1297766

25. Woodward, D. E., Tyson, R., Myerscough, M., R., Murray, J. D., Budrene, E. O., \& Berg, H. C. Spatio-temporal patterns generated by Salmonella typhimurium. Biophys. 68 (1995), 2181-2189. 